\title{
COMPUTATION OF THE ZEROS OF $p$-ADIC $L$-FUNCTIONS
}

\author{
R. ERNVALL AND T. METSÄNKYLÄ
}

\begin{abstract}
ABSTRACr. The authors have computed the zeros of the Kubota-Leopoldt $p$ adic $L$-functions $L_{p}(s, \chi)$ for some small odd primes $p$ and for a number of Dirichlet characters $\chi$. The zeros of the corresponding Iwasawa power series $f_{\theta}(T)$ are also computed. The characters $\chi$ (associated with quadratic extensions of the $p$ th cyclotomic field) are chosen so as to cover as many different splitting types of $f_{\theta}(T)$ as possible. The $\lambda$-invariant of this power series, equal to its number of zeros, assumes values up to 8 .

The article is a report on these computations and their results, including the required theoretical background. Much effort is devoted to a study of the accuracy of the computed approximations.
\end{abstract}

\section{INTRODUCTION}

This article is a report on our computations of the zeros of the KubotaLeopoldt $p$-adic $L$-functions $L_{p}(s, \chi)$, where $p$ is an odd prime and $\chi$ a Dirichlet character. Some theoretical results about the zeros are also established.

Let $f_{\theta}(T)$ denote the Iwasawa power series representing $L_{p}(s, \chi)$; here $\theta$, the so-called first factor of $\chi$ (for $p$ ), is assumed to be nonprincipal. A major part of our work consists in computing the zeros of $f_{\theta}(T)$. These, besides being of interest in their own right, have a close relationship to the zeros of $L_{p}(s, \chi)$. They are both theoretically and computationally easier to treat than the latter; in particular, their number equals $\lambda_{\theta}$, the $\lambda$-invariant of the power series.

Several mathematicians have previously computed zeros of $L_{p}(s, \theta)$ and $f_{\theta}(T)$; see $[7,10,11,12,13]$. Our computations followed the same main lines as those by Wagstaff in [13]. However, apart from a few examples (in [13]), all the earlier computations deal with the case $\lambda_{\theta}=1$, whereas we let $\lambda_{\theta}$ vary from 2 to 6 . We also have partial results with $\lambda_{\theta}=7$ and 8 . The characters $\theta$ we used in the computations are mainly selected from our recent work [3]. They turned out to represent quite a great variety of different cases, as regards the splitting type and splitting field of $f_{\theta}(T)$. It was our intention to elaborate a systematic approach applicable to all of these cases, including a thorough study of the accuracy of the computed approximations.

In the material computed by us there are also many examples leading to zeros of $L_{p}(s, \chi)$ for characters $\chi \neq \theta$, i.e., for $\chi$ not of the "first kind". These

Received July 3, 1990; revised March 8, 1991.

1991 Mathematics Subject Classification. Primary 11S40, 11Y05, 11Y40, 11R20, 11 R23.

Key words and phrases. p-adic $L$-functions, computation of zeros, factorization of polynomials, Newton's tangent method, Abelian fields, Iwasawa theory. 
results, as well as some other results pertaining to more complicated cases, will be presented in the forthcoming Part II of the article.

The computations for the present research were performed by the first author. The second author did most of the theoretical work while spending the academic year 1988-89 at the Ohio State University in Columbus, Ohio. He would like to thank the Department of Mathematics at the OSU for its hospitality and the Academy of Finland for financial support.

\section{Preliminaries}

For an odd prime $p$ let us fix an embedding of the field of algebraic numbers into $\mathbb{C}_{p}$, the completion of an algebraic closure of the $p$-adic field $\mathbb{Q}_{p}$. Let $v_{p}$ denote the $p$-adic exponential valuation on $\mathbb{C}_{p}$, normalized so that $v_{p}(p)=1$.

The $p$-adic $L$-function $L_{p}(s, \chi)$ attached to a Dirichlet character $\chi$, always assumed primitive, was first introduced by Kubota and Leopoldt in [9]. Recall that $L_{p}(s, \chi)$ is a meromorphic function on

$$
D_{s}=\left\{s \in \mathbb{C}_{p}: v_{p}(s)>-1+\frac{1}{p-1}\right\}
$$

satisfying

$$
L_{p}(1-m, \chi)=\left(1-\chi_{m}(p) p^{m-1}\right) L\left(1-m, \chi_{m}\right) \quad(m=1,2, \ldots),
$$

where $L(s, \chi)$ denotes the Dirichlet $L$-function and $\chi_{m}=\chi \omega^{-m}, \omega$ being the Teichmüller character mod $p$. In fact, $L_{p}(s, \chi)$ is analytic on $D_{s}$, except that there is a pole at $s=1$ when $\chi=1$. We assume that $\chi$ is even, i.e., $\chi(-1)=1$, since otherwise $L_{p}(s, \chi)$ vanishes identically.

As in the Introduction, let $\theta$ be the first factor of $\chi$. Write $\chi=\theta \psi_{n}$, where $\psi_{n}$ is of order $p^{n} \quad(n \geq 0)$. Note that the conductor of $\theta$ is of the form $d$ or $d p$ with $d$ prime to $p$, and the conductor of $\psi_{n}$ equals $p^{n+1}$ (or 1 if $\left.n=0\right)$.

Let $\mathscr{O}_{\theta}$ denote the ring of integers in the extension of $\mathbb{Q}_{p}$ generated by all the values of $\theta$. If $\theta$ is nonprincipal, there is a power series

$$
f_{\theta}(T)=\sum_{j=0}^{\infty} a_{j} T^{j} \in \mathscr{O}_{\theta}[[T]]
$$

such that

$$
L_{p}(s, \chi)=f_{\theta}\left(\rho_{n}(1+d p)^{s}-1\right)
$$

with $\rho_{n}=\psi_{n}(1+d p)^{-1}$. For $\theta=1, f_{\theta}(T)$ is to be replaced by a quotient of two power series.

Assume that $\theta \neq 1$. Viewing $f_{\theta}(T)$ as a function of $T$ we see that it analytic on the open unit disc

$$
D_{T}=\left\{T \in \mathbb{C}_{p}: v_{p}(T)>0\right\} .
$$

By the Ferrero-Washington theorem, $v_{p}\left(a_{j}\right)$ vanishes for some $j \geq 0$. Thus the $p$-adic Weierstrass preparation theorem enables one to write

$$
f_{\theta}(T)=u_{\theta}(T)\left(b_{0}+b_{1} T+\cdots+b_{\lambda-1} T^{\lambda-1}+T^{\lambda}\right),
$$

where $u_{\theta}(T)$ is an invertible power series in $\mathscr{O}_{\theta}[[T]]$, the coefficients $b_{j}$ belong to the maximal ideal of $\mathscr{O}_{\theta}$, and $\lambda=\lambda_{\theta}$ is the $\lambda$-invariant of $f_{\theta}(T)$,

$$
\lambda_{\theta}=\min \left\{j \geq 0: v_{p}\left(a_{j}\right)=0\right\} \text {. }
$$


From this it follows that $f_{\theta}(T)$ has exactly $\lambda$ zeros $T_{1}, \ldots, T_{\lambda}$ in $D_{T}$ (not necessarily distinct).

If $\theta=1$, then

$$
f_{\theta}(T)=\frac{g_{\theta}(T)}{1-(1+p) /(1+T)}
$$

with $g_{\theta}(T) \in \mathscr{O}_{\theta}[[T]], g_{\theta}(T)$ invertible (e.g., $[14$, p. 125]). This formal identity of power series indeed gives an equation in $\mathbb{C}_{p}$ for every $T \in D_{T}$ except for $T=p$ (corresponding to the pole of $L_{p}(s, 1)$ ). Consequently, $f_{\theta}(T)$ has no zeros for $\theta=1$, and also $L_{p}\left(s, \psi_{n}\right)$ is nonzero in $D_{s}$.

Henceforth we will exclude the case $\theta=1$.

To determine the zeros of $L_{p}(s, \chi)$, we first compute the $\lambda$ zeros of $f_{\theta}(T)$. Hence, one has to understand the relationship between the zeros of these functions. This will be discussed in the next section.

\section{Relationship Between the ZeRos of $L_{p}$ AND $f_{\theta}$}

The results of this section are partly due to Childress and Gold [2]. Our presentation is somewhat different, however.

By (2.1), the mapping

$$
\kappa_{\chi}: D_{s} \rightarrow D_{T}, \quad \kappa_{\chi}(s)=\rho_{n}(1+d p)^{s}-1
$$

sends any zero of $L_{p}(s, \chi)$ to a zero of $f_{\theta}(T)$. To study $\kappa_{\chi}$ more closely, set

$$
\begin{gathered}
D_{T}^{0}=\left\{T \in \mathbb{C}_{p}: v_{p}(T)>\frac{1}{p-1}\right\}, \\
C_{n}=\left\{T \in \mathbb{C}_{p}: v_{p}(T)=\frac{1}{(p-1) p^{n-1}}\right\} \quad(n=1,2, \ldots) .
\end{gathered}
$$

Proposition 1. Let $\chi=\theta \psi_{n}$. If $s \in D_{s}$, then

$$
T=\kappa_{\chi}(s) \in \begin{cases}D_{T}^{0} & \text { for } n=0, \\ C_{n} & \text { for } n \geq 1 .\end{cases}
$$

Proof. Introducing the $p$-adic exponential and logarithm functions, we may write

$$
T=\rho_{n}(\exp (s \log (1+d p))-1)+\rho_{n}-1 .
$$

Since

$$
\begin{gathered}
v_{p}(\exp (s \log (1+d p))-1)=v_{p}(s \log (1+d p))=v_{p}(s)+1>\frac{1}{p-1}, \\
v_{p}\left(\rho_{n}-1\right)=\frac{1}{(p-1) p^{n-1}} \quad(n=1,2, \ldots),
\end{gathered}
$$

and since $\rho_{0}=1$, the assertion follows.

Let $T_{0}$ be a zero of $f_{\theta}(T)$ in $D_{T}$. If there exists a character $\chi=\theta \psi_{n}$ and a zero $s_{0}$ of $L_{p}(s, \chi)$ such that $\kappa_{\chi}\left(s_{0}\right)=T_{0}$, we say that the zero $s_{0}$-or, more precisely, the pair $\left(\psi_{n}, s_{0}\right)$-corresponds to $T_{0}$. As observed by Washington [15, p. 351], such a pair $\left(\psi_{n}, s_{0}\right)$, if it exists, is unique. Indeed, an equation of the form

$$
\rho_{n_{1}}(1+d p)^{s_{1}}=\rho_{n_{2}}(1+d p)^{s_{2}}
$$


implies, upon raising to the $p^{n_{1}+n_{2}}$ th power, that $s_{1}=s_{2}$. Hence, moreover, $\rho_{n_{1}}=\rho_{n_{2}}$, and so $\psi_{n_{1}}=\psi_{n_{2}}$.

By Proposition 1, a necessary condition for the existence of a zero $s_{0}$ corresponding to $T_{0}$ is that $T_{0}$ lies in $D_{T}^{0}$ or on one of the $C_{n}$. The next propositions provide some sufficient conditions.

Remark. In [2] it is shown that there always exist zeros of more general $L$ functions corresponding to $T_{0}$ (in the above sense). These functions, $p$-adic $L$-functions over totally real fields, are not discussed in the present work.

Proposition 2. If $f_{\theta}\left(T_{0}\right)=0$ and $T_{0} \in D_{T}^{0}$, then there is a zero $s_{0}$ of $L_{p}(s, \theta)$ corresponding to $T_{0}$. In fact,

$$
s_{0}=\frac{\log \left(1+T_{0}\right)}{\log (1+d p)}
$$

Proof. Properties of the exp and log function imply that the mapping $\kappa_{\theta}: D_{s} \rightarrow$ $D_{T}^{0}$ has the inverse

$$
\kappa_{\theta}^{-1}: D_{T}^{0} \rightarrow D_{s}, \quad \kappa_{\theta}^{-1}(T)=\frac{\log (1+T)}{\log (1+d p)} .
$$

Proposition 3. Let $T_{0} \in C_{n}$ be a zero of $f_{\theta}(T)$. There is a zero $s_{0}$ of some $L_{p}\left(s, \theta \psi_{n}\right)$ corresponding to $T_{0}$ if and only if $\log \left(1+T_{0}\right) / \log (1+d p) \in D_{s}$. If this is the case, then $\psi_{n}$ is determined by

$$
\rho_{n}=\psi_{n}(1+d p)^{-1}, \quad v_{p}\left(1-\rho_{n}+T_{0}\right)>\frac{1}{p-1},
$$

and $s_{0}$ is given by (3.2).

Proof. If $s_{0}$ corresponds to $T_{0}$, it follows from the equation $T_{0}=\rho_{n}(1+d p)^{s_{0}}-$ 1 that $s_{0}$ satisfies (3.2). Thus, in particular, $\log \left(1+T_{0}\right) / \log (1+d p) \in D_{s}$.

Conversely, assume that $s_{0}=\log \left(1+T_{0}\right) / \log (1+d p) \in D_{s}$. Then

$$
\log \left((1+d p)^{s_{0}}\right)=s_{0} \log (1+d p)=\log \left(1+T_{0}\right),
$$

and so, since $(1+d p)^{s_{0}}$ and $1+T_{0}$ are $p$-adic units,

$$
1+T_{0}=\zeta(1+d p)^{s_{0}} \quad(\zeta \text { a root of } 1) .
$$

By writing this in the form

$$
1-\zeta+T_{0}=\zeta\left((1+d p)^{s_{0}}-1\right)
$$

and by using the fact that $T_{0} \in C_{n}$, we find (cf. the proof of Proposition 1) that $v_{p}(1-\zeta)=v_{p}\left(T_{0}\right)=1 /(p-1) p^{n-1}$. Consequently, $\zeta$ is of order $p^{n}$, and we may set $\zeta=\rho_{n}=\psi_{n}(1+d p)^{-1}$ for some character $\psi_{n}$. By (3.4), $L_{p}\left(s_{0}, \theta \psi_{n}\right)=f_{\theta}\left(T_{0}\right)=0$. Moreover, by (3.5),

$$
v_{p}\left(1-\rho_{n}+T_{0}\right)=v_{p}\left((1+d p)^{s_{0}}-1\right)>\frac{1}{p-1} .
$$

It remains to show that the inequality in (3.3) determines $\rho_{n}$ uniquely. Let $\rho_{n}^{\prime}$ be any root of unity with $p$-power order such that $v_{p}\left(1-\rho_{n}^{\prime}+T_{0}\right)>1 /(p-1)$. Then $v_{p}\left(\rho_{n}-\rho_{n}^{\prime}\right)>1 /(p-1)$. Therefore, $\rho_{n}^{\prime}=\rho_{n}$.

With the assumption of Proposition 3, when does $\log \left(1+T_{0}\right) / \log (1+d p)$ lie in $D_{s}$ ? Some results about this are presented in the following Propositions 4 and 5. 
For the rest of this section we suppose that $T_{0} \in C_{n}$ is a zero of $f_{\theta}(T)$ and $s_{0}$ is given by (3.2). Let $\zeta_{m}$ denote a primitive $m$ th root of 1 .

Proposition 4. If $s_{0} \in D_{s}$, then $\zeta_{p^{n}} \in \mathbb{Q}_{p}\left(T_{0}\right)$.

Proof. Since $\rho_{n}$ is a primitive $p^{n}$ th root of 1 , its conjugates $\rho$ satisfy $v_{p}\left(\rho-\rho_{n}\right)$ $=v_{p}\left(1-\rho^{-1} \rho_{n}\right) \leq 1 /(p-1)$ whenever $\rho \neq \rho_{n}$. Hence the inequality in (3.3) yields by Krasner's lemma that $\rho_{n} \in \mathbb{Q}_{p}\left(1+T_{0}\right)$.

Proposition 5. (i) Let $\theta$ satisfy $\mathscr{O}_{\theta}=\mathbb{Z}_{p}$ and $\lambda_{\theta}=(p-1) p^{n-1}$. If $s_{0} \in D_{s}$, then $T_{0} \in \mathbb{Q}_{p}\left(\zeta_{p^{n}}\right)$.

(ii) Conversely, if $n=1$ and $T_{0} \in \mathbb{Q}_{p}\left(\zeta_{p}\right)$, then $s_{0} \in D_{s}$.

Proof. (i) Recall by (2.2) that $T_{0}$ is a zero of a polynomial in $\mathbb{Z}_{p}[T]$ of degree $(p-1) p^{n-1}$. Thus, if $s_{0} \in D_{s}$, it follows from Proposition 4 that $\mathbb{Q}_{p}\left(T_{0}\right)=$ $\mathbb{Q}_{p}\left(\zeta_{p^{n}}\right)$.

(ii) Suppose that $v_{p}\left(T_{0}\right)=1 /(p-1)$ and $T_{0} \in \mathbb{Q}_{p}\left(\zeta_{p}\right)$, say

$$
T_{0} \equiv a \pi \quad\left(\bmod \pi^{2}\right) \quad(a \in \mathbb{Z} \backslash p \mathbb{Z}),
$$

where $\pi=1-\zeta_{p}$. We have $p=\varepsilon \pi^{p-1}$ with a $p$-adic unit $\varepsilon$,

$$
\varepsilon=\prod_{k=1}^{p-1} \frac{1-\zeta_{p}^{k}}{1-\zeta_{p}} \equiv \prod_{k=1}^{p-1} k \equiv-1 \quad(\bmod \pi)
$$

Consequently,

$$
\log \left(1+T_{0}\right) \equiv T_{0}+\frac{T_{0}^{p}}{p} \equiv a \pi+\frac{a^{p} \pi}{\varepsilon} \equiv\left(a-a^{p}\right) \pi \equiv 0 \quad\left(\bmod \pi^{2}\right),
$$

and so $v_{p}\left(s_{0}\right)=v_{p}\left(\log \left(1+T_{0}\right)\right)-1>1 /(p-1)-1$.

With this in mind, a first interesting open question is whether Proposition 5(ii) can be generalized to $n>1$. In the numerical material so far generated by us there are only few zeros $T_{0}$ lying in some $C_{n}$ for $n>1$. These are examples with $p=3, n=2$, and the zeros $T_{0}$ never belong to $\mathbb{Q}_{3}\left(\zeta_{9}\right)$.

\section{Plan of the computation}

Tables of zeros of $f_{\theta}(T)$ and $L_{p}(s, \theta)$ were first published by Iwasawa and Sims [7] and by Wagstaff [12] who were concerned with $\theta=\omega^{t}$, a power of the Teichmüller character mod $p$, for a number of irregular primes $p$. Sunseri [11] expanded these results by computing considerably more places for the zeros up to $p<1000$.

In [13] Wagstaff reported on new computations with $\theta=\theta_{m}$, the quadratic character of the field $\mathbb{Q}(\sqrt{m})$, for several positive primes $m \equiv 1(\bmod 4)$ and for $p=3$ and 5 . His results were partly extended by Lamprecht and Zimmer [10] to $p \leq 13$. Also, [15] contains some interesting data about the zeros of $L_{3}\left(s, \theta_{m}\right)$.

With a few exceptions in [13], all the above results are about the case $\lambda_{\theta}=1$. Hence, for the characters $\theta$ in question, there is always a unique zero $T_{0} \in p \mathbb{Z}_{p}$ of $f_{\theta}(T)$ and a zero $s_{0}$ of $L_{p}(s, \theta)$ corresponding to $T_{0}$. Moreover, in all cases one has $v_{p}\left(T_{0}\right)=1$ and, thus, $v_{p}\left(s_{0}\right)=0$. 
In the present work we are concerned with (even) characters

$$
\theta=\theta_{m} \omega^{t} \quad(p \nmid m, 0 \leq t \leq p-2)
$$

for $p=3,5,7$, and 11, allowing both positive and negative values, not necessarily prime, for $m$. For characters of this form, values of $\lambda_{\theta}$ were computed in [3]. We selected examples with $\lambda_{\theta}>1$, trying to find as many different types of cases as possible. The computation procedure was organized to follow the same main lines as that in [13]. Thus, there were the following four principal steps:

(1) computing $L_{p}(s, \theta)$ as a power series in $s$ from Washington's formula (see (5.1) below),

(2) converting this function by (2.1) into the power series $f_{\theta}(T)$,

(3) computing the zeros of $f_{\theta}(T)$ by Newton's tangent method, and

(4) converting these zeros to the zeros of $L_{p}\left(s, \theta \psi_{n}\right)$ according to Propositions 2 and 3.

Note that $f_{\theta}(T)$ is not canonically defined. Following Washington, we adopt definition (2.1) which is quite natural, while [13] and [10] define

$$
L_{p}(s, \theta)=-f_{\theta}\left((1+p)^{-s}-1\right) \text {. }
$$

The $p$-adic values of the coefficients and of the zeros of $f_{\theta}(T)$ of course remain invariant.

We performed several additional computations to check the results at each step. For example, when there were zeros $s_{0}$ of $L_{p}\left(s, \theta \psi_{n}\right)$ with $\psi_{n} \neq 1$ (which in fact happened for $p=3$ and $n=1$ only), we also computed this $L$-function to verify the vanishing of $L_{p}\left(s_{0}, \theta \psi_{n}\right)$.

To come up with a representative sample of examples, we divided the computing process into two parts. First, in Program A, all characters $\theta$ with $\lambda_{\theta}>1$ from our earlier work [3] were run through the steps (1) and (2). Here, the power series were computed just to a few places of the very first coefficients. In most cases this was sufficient to determine the $p$-adic values of the zeros of $f_{\theta}(T)$; if it was not, the computations were repeated with a greater number of places.

In Program B, we performed the entire procedure (1)-(4) for characters chosen on the basis of Program A. Some examples were also taken from Kobayashi [8]. The number of computed places varied case by case depending on the type of the example. We point out that step (1) takes much more time to execute than the other steps, and the time grows rapidly when the number of places is increased.

Program B contains cases with $\lambda_{\theta}$ up to 6. From Program A we also have some results about a few cases in which $\lambda_{\theta}=7$ or 8 .

The main part of the computations was carried out in 1988-89 on an IBM 3033 computer at the University of Turku.

A detailed description of the computation procedure appears in the following sections. In $\S \S 5-9$ problems that arose at each step are studied from a theoretical point of view, while $\S 11$ in the Supplements section at the end of this issue gives more information about the actual computations. Results are presented in $\S 10$. Finally, $\S 12$ of the Supplements section is devoted to the proofs of some propositions of a more technical nature. 
Henceforth in this paper, we let $\theta$ denote a nonprincipal character of the form $\theta_{m} \omega^{t}$.

\section{Computing A $p$-ADIC $L$-FUnCtion}

Washington's formula $[14$, p. 57$]$ reads

$$
L_{p}(s, \chi)=\frac{1}{s-1} \frac{1}{\Phi} \sum_{\substack{a=1 \\
p \nmid a}}^{\Phi} \chi(a)\langle a\rangle^{1-s} \sum_{j=0}^{\infty}\left(\begin{array}{c}
1-s \\
j
\end{array}\right) B_{j}\left(\frac{\Phi}{a}\right)^{j},
$$

where $\Phi$ is any common multiple of $p$ and the conductor of $\chi$; the notation $\langle a\rangle$ stands for $a \omega^{-1}(a)$, and $B_{j}$ denotes the $j$ th Bernoulli number (with $B_{1}=$ $-1 / 2)$. When using this formula for the computation of $L_{p}(s, \theta)$, the optimal choice of $\Phi$ is

$$
\Phi=d p \quad \text { with } d=\text { conductor of } \theta_{m} \text { (either }|m| \text { or } 4|m| \text { ). }
$$

As in [13], expand the right-hand side of (5.1) in a power series of $s$ by writing

$$
\langle a\rangle^{1-s}=\sum_{\nu=0}^{\infty}\left(\begin{array}{c}
1-s \\
\nu
\end{array}\right)(\langle a\rangle-1)^{\nu}
$$

and expanding the binomial coefficients in powers of $s$. This will be discussed more closely in $\S 11$ of the Supplements section. As a result, one obtains

$$
L_{p}(s, \theta)=\sum_{i=0}^{\infty} u_{i} s^{i}
$$

with coefficients $u_{i}$ in $\mathbb{Q}_{p}$. It follows from [14, Theorem 5.12] (see also Proposition 6 below) that in fact $u_{i} \in \mathbb{Z}_{p}$.

By the computation of $L_{p}(s, \theta)$ we mean the calculation of rational integers $\bar{u}_{i}$ such that

$$
u_{i} \equiv \bar{u}_{i} \quad\left(\bmod p^{M}\right) \quad \text { for } i=0, \ldots, i_{M},
$$

where $M$ is a suitably chosen integer and $i_{M}$ satisfies

$$
i_{M} \geq M-1, \quad u_{i} \equiv 0 \quad\left(\bmod p^{M}\right) \text { for all } i>i_{M} .
$$

To obtain a value for $i_{M}$, we need a lower bound for $v_{p}\left(u_{i}\right)$. The following estimate is sharper and more convenient than those given in [13] and [10].

Proposition 6. We have

$$
v_{p}\left(u_{i}\right) \geq i-\frac{i-1}{p-1} \quad(i=1,2, \ldots) .
$$

Proof. The idea is to compare the expansion in (5.2) with that deriving from (2.1). First observe that

$$
(1+d p)^{s}-1=\exp (s \log (1+d p))-1=\sum_{i=1}^{\infty} d_{i} s^{i}
$$

with $d_{i}=(\log (1+d p))^{i} / i$ !. We have

$$
v_{p}\left(d_{i}\right)=i-v_{p}(i !) \geq i-\frac{i-1}{p-1} .
$$


Hence,

$$
v_{p}\left(d_{i} s^{i}\right)>v_{p}\left(d_{i}\right)+i\left(-1+\frac{1}{p-1}\right)>0
$$

for all $s \in D_{s}$, and so a general principle (e.g., [1, p. 284]) allows us to write the composite function

$$
f_{\theta}\left((1+d p)^{s}-1\right)=\sum_{j=0}^{\infty} a_{j}\left(\sum_{i=1}^{\infty} d_{i} s^{i}\right)^{j}
$$

formally as a power series in $s$. Compared to (5.2), this implies that $u_{0}=a_{0}$ and

$$
u_{i}=\sum_{j=1}^{i} a_{j} d_{i j} \quad \text { with } \quad d_{i j}=\sum_{t_{1}+\cdots+t_{j}=i} d_{t_{1}} \cdots d_{t_{j}} \quad(i=1,2, \ldots) .
$$

Since $v_{p}\left(a_{j}\right) \geq 0$, we have to check that $v_{p}\left(d_{i j}\right) \geq i-(i-1) /(p-1)$ for $j=1, \ldots, i$. In view of $(5.5)$ this is indeed the case.

Remark. An analogous result for the coefficients in the expansion of $L_{p}\left(s, \theta \psi_{n}\right)$ will be proved in Part II.

\section{Computing the Iwasawa POWER SERIES}

If $T \in D_{T}^{0}$, then $\log (1+T) / \log (1+d p) \in D_{s}$ and we have, by $(2.1)$,

$$
f_{\theta}(T)=L_{p}\left(\frac{\log (1+T)}{\log (1+d p)}, \theta\right) .
$$

We now obtain the coefficients of the power series $f_{\theta}(T)=\sum_{j=0}^{\infty} a_{j} T^{j}$ from this formula by using our expansion (5.2) for $L_{p}(s, \theta)$. Write

$$
\frac{\log (1+T)}{\log (1+d p)}=\frac{1}{p} \sum_{j=1}^{\infty} e_{j} T^{j} \quad \text { with } \quad e_{j}=\frac{(-1)^{j-1}}{j} \frac{p}{\log (1+d p)},
$$

and note that $v_{p}\left(p^{-1} e_{j} T^{j}\right)>-1+1 /(p-1)$ for all $j \geq 1$, provided $v_{p}(T)>$ $v_{0}$, where $v_{0}$ is a suitable constant, $v_{0} \geq 1 /(p-1)$. Consequently, by the substitution principle employed in the proof of Proposition 6, we find that

$$
L_{p}\left(\frac{\log (1+T)}{\log (1+d p)}, \theta\right)=\sum_{i=0}^{\infty} u_{i}\left(\frac{1}{p} \sum_{j=1}^{\infty} e_{j} T^{j}\right)^{i}=\sum_{j=0}^{\infty} a_{j}^{\prime} T^{j},
$$

say, where $a_{0}^{\prime}=u_{0}$ and

$$
a_{j}^{\prime}=\frac{1}{p^{j}} \sum_{i=1}^{j} p^{j-i} u_{i} \sum_{t_{1}+\cdots+t_{i}=j} e_{t_{1}} \cdots e_{t_{i}} \quad(j=1,2, \ldots) .
$$

Comparison with (6.1) now yields (and here the restriction $v_{p}(T)>v_{0}$ becomes superfluous)

$$
a_{j}=a_{j}^{\prime} \quad(j=0,1, \ldots) .
$$

As mentioned in $\S 2$, the $a_{j}$ are $p$-adic integers. Our computation of $f_{\theta}(T)$ consists of the calculation of rational integers $\bar{a}_{j}$ approximating $a_{j}$ for $j=$ 
$0, \ldots, M-1$. We do this by using (6.3), where $u_{i}$ is replaced by its approximation $\bar{u}_{i} \bmod p^{M}$ as described in $\S 5$, and similarly $e_{j}$ by an approximation $\bar{e}_{j}$ $\bmod p^{M}$. Note that, in contrast to $u_{i}$, the numbers $e_{j}$ do not tend ( $p$-adically) to zero; in fact, $e_{j}$ is generally not even a $p$-adic integer. By the congruence $e_{j} \equiv \bar{e}_{j}\left(\bmod p^{M}\right)$ we mean that $v_{p}\left(e_{j}-\bar{e}_{j}\right) \geq M$.

For the approximations $\bar{a}_{j}$ obtained in this way we may formulate the following result. The proof is postponed to $\S 12$ (see Supplements section).

Proposition 7. We have $a_{j} \equiv \bar{a}_{j}\left(\bmod p^{M-j}\right)$ for $j=0, \ldots, M-1$.

\section{COMPUTING ZEROS OF A POWER SERIES}

Let

$$
f(T)=\sum_{j=0}^{\infty} c_{j} T^{j} \in \mathscr{O}_{F}[[T]],
$$

where $\mathscr{O}_{F}$ is the integer ring of $F$, a finite extension of $\mathbb{Q}_{p}$ (all extensions of $\mathbb{Q}_{p}$ are considered in $\mathbb{C}_{p}$ ). We will study the problem of computing zeros of $f(T)$, viewed as a function of $T$ on $D_{T}$. This is then applied to the case when $f(T)$ is either $f_{\theta}(T)$ or the quotient of $f_{\theta}(T)$ by some of its linear factors.

Suppose we know that there is a simple zero $T_{0} \in D_{T}$ in an extension $E$ of $F$. Then $T_{0} \in \pi \mathscr{O}_{E}$, where $\pi$ is a prime element of $\mathscr{O}_{E}$. We let $v_{\pi}$ denote the valuation on $\mathbb{C}_{p}$ to the base $\pi$, that is, $v_{\pi}=e v_{p}$, where $e$ is the ramification index of $E / \mathbb{Q}_{p}$. Note that $v_{\pi}\left(f^{\prime}\left(T_{0}\right)\right)$ is a nonnegative integer ( $f^{\prime}$ stands for the derivative of $f$ ).

Once a sufficiently good initial approximation for $T_{0}$ is known, one can compute $T_{0}$ to any desired accuracy by using Newton's tangent method. This is based on the following result.

Proposition 8 (Newton algorithm). Let $t_{0} \in \pi \mathscr{O}_{E}$ satisfy $f\left(t_{0}\right) \equiv 0\left(\bmod \pi^{2 \gamma+1}\right)$, where $\gamma=v_{\pi}\left(f^{\prime}\left(t_{0}\right)\right)$. Then there exists a unique $T_{0} \in \pi \mathscr{O}_{E}$ such that

$$
f\left(T_{0}\right)=0, \quad T_{0} \equiv t_{0} \quad\left(\bmod \pi^{\gamma+1}\right) .
$$

In fact, $T_{0}=\lim _{n \rightarrow \infty} t_{n}$, where $t_{n}$ is defined by

$$
t_{n}=t_{n-1}-\frac{f\left(t_{n-1}\right)}{f^{\prime}\left(t_{n-1}\right)} \quad(n=1,2, \ldots) .
$$

Proof. For the uniqueness which is crucial in the present work, one can give the following simple proof (omitted in several standard references).

Write $f(T)=\left(T-T_{0}\right) g(T)$ with $g(T) \in \mathbb{C}_{p}[[T]]$. Since $g\left(T_{0}\right)=f^{\prime}\left(T_{0}\right)$, we have $\gamma=v_{\pi}\left(g\left(T_{0}\right)\right)=v_{\pi}\left(g\left(t_{0}\right)\right)$. Thus, if $U_{0}$ is any zero congruent to $t_{0}\left(\bmod \pi^{\gamma+1}\right)$, we see that $v_{\pi}\left(g\left(U_{0}\right)\right)=\gamma$ and so $g\left(U_{0}\right) \neq 0$. But $\left(U_{0}-T_{0}\right) g\left(U_{0}\right)=f\left(U_{0}\right)=0$, hence $U_{0}=T_{0}$.

From the well-known existence proof we just quote the following formulas, valid for all $n \geq 1$ :

$$
\begin{gathered}
t_{n} \equiv t_{n-1} \quad\left(\bmod \pi^{\gamma+n}\right) \\
f\left(t_{n}\right) \equiv 0 \quad\left(\bmod \pi^{2 \gamma+n+1}\right), \\
v_{\pi}\left(f^{\prime}\left(t_{n}\right)\right)=v_{\pi}\left(f^{\prime}\left(T_{0}\right)\right)=\gamma
\end{gathered}
$$


As regards the accuracy of the approximation $t_{n},(7.1)$ shows that

$$
T_{0} \equiv t_{n} \quad\left(\bmod \pi^{\gamma+n+1}\right)
$$

In our real situation, the power series $f(T)$ is given approximately, in fact, as a polynomial. However, its zeros $T_{0}$ are approximately the same as the zeros of this polynomial because $T_{0}^{j} \rightarrow 0$ as $j \rightarrow \infty$. We formulate this in a quantitative form.

Let $\gamma$ and $t_{n}(n \geq 0)$ be as in Proposition 8. Fix $N \in \mathbb{Z}$ so that $N>\gamma$. There is a polynomial

$$
\bar{f}(T)=\sum_{j=0}^{N^{\prime}} \bar{c}_{j} T^{j} \in \mathscr{O}_{F}[T]
$$

with the following properties:

(i) $v_{\pi}\left(\bar{f}^{\prime}\left(t_{0}\right)\right)=\gamma, \bar{f}\left(t_{0}\right) \equiv 0\left(\bmod \pi^{2 \gamma+1}\right)$;

(ii) if $\bar{t}_{0}, \bar{t}_{1}, \ldots$ is the sequence obtained for $\bar{f}(T)$ by the Newton algorithm, with the initial approximation $\bar{t}_{0}=t_{0}$ and each $\bar{t}_{n}$ computed mod $\pi^{\gamma+n+1}$, then the zero $T_{0}=\lim _{n \rightarrow \infty} t_{n}$ of $f(T)$ satisfies

$$
T_{0} \equiv \bar{t}_{N-\gamma} \quad\left(\bmod \pi^{N+1}\right) \text {. }
$$

In fact, such a polynomial $\bar{f}(T)$ is given by the following proposition, which will be proved in $\S 12$ of the Supplements section.

Proposition 9. Choose $w \in \mathbb{Z}$ so that $1 \leq w \leq \gamma+1$ and $t_{0} \equiv 0\left(\bmod \pi^{w}\right)$. Let $N^{\prime}=[(N+\gamma) / w]$, where $[x]$ denotes the largest integer $\leq x$, and assume that $\bar{c}_{j} \in \mathscr{O}_{F}$ satisfies

$$
c_{j} \equiv \bar{c}_{j} \quad\left(\bmod \pi^{N+\gamma+1-w j}\right) \quad\left(j=0, \ldots, N^{\prime}\right) .
$$

Then the polynomial $\bar{f}(T)$ defined in (7.5) has the properties (i) and (ii).

\section{COMPUTING ZeRos of $f_{\theta}$ AND $L_{p}$}

Now consider the Iwasawa power series $f_{\theta}(T)=\sum_{j=0}^{\infty} a_{j} T^{j} \in \mathbb{Z}_{p}[[T]]$. In view of Proposition 7, we will assume that the $a_{j}$ are approximated by $\bar{a}_{j} \in \mathbb{Z}$, so that $a_{j} \equiv \bar{a}_{j}\left(\bmod p^{M-j}\right)$. Set

$$
\bar{f}_{\theta}(T)=\sum_{j=0}^{M-1} \bar{a}_{j} T^{j} .
$$

Let $T_{0}$ be a simple zero of $f_{\theta}(T)$. As in the previous section, let $E$ denote an extension of $\mathbb{Q}_{p}$ containing $T_{0}$; also keep the notations $\pi$ and $e$ associated with $E$.

Proposition 10. Suppose that $t_{0} \in \pi^{c} \mathscr{O}_{E} \quad(c \geq 1)$ satisfies

$$
T_{0} \equiv t_{0} \quad\left(\bmod \pi^{\gamma+1}\right), \quad f_{\theta}\left(t_{0}\right) \equiv 0 \quad\left(\bmod \pi^{2 \gamma+1}\right),
$$

where $\gamma=v_{\pi}\left(f_{\theta}^{\prime}\left(t_{0}\right)\right)$. The Newton algorithm applied to $\bar{f}_{\theta}(T)$, with $t_{0}$ as the initial approximation, produces a zero $\bar{T}_{0}$ satisfying

$$
T_{0} \equiv \bar{T}_{0} \quad\left(\bmod \pi^{\rho M-\gamma}\right)
$$


with $\rho=\min (e, c)$, provided that $\rho \leq \gamma+1$ and $\rho M>2 \gamma+1$.

Proof. Apply Proposition 9 with $N=\rho M-\gamma-1$ and $w=\rho$, observing that $a_{j} \equiv \bar{a}_{j}\left(\bmod \pi^{\rho M-\rho j}\right)$. The claimed congruence follows from (7.6).

Remarks. The last two inequalities in Proposition 10 are fulfilled whenever $c$ and $M$ are chosen appropriately. Hence, there remains the question how to find a field $E$ containing a zero $T_{0}$ and how to determine an initial approximation $t_{0}$. This will be discussed in the next section.

Once one zero $T_{0}$ of $f_{\theta}(T)$ has been computed, it may be better to deal with $f_{\theta}(T) /\left(T-T_{0}\right)$ when calculating further zeros. This is the case, in particular, if $T_{0}=0$, but sometimes also for other $T_{0} \in \mathbb{Q}_{p}$. The above congruences are then to be modified slightly.

Our final task is to determine the zeros $s_{0}$ of $L_{p}\left(s, \theta \psi_{n}\right)$ which correspond to each $T_{0}$ lying in $D_{T}^{0}$ or on some $C_{n} \quad(n \geq 1)$. By Propositions 2 and 3, and by (6.2), $s_{0}$ is obtained from

$$
s_{0}=\frac{\log \left(1+T_{0}\right)}{\log (1+d p)}=\frac{1}{p} \sum_{j=1}^{\infty} e_{j} T_{0}^{j}
$$

in the case $T_{0} \in C_{n}$ one moreover has to check that $s_{0} \in D_{s}$. Note that the field $E$ containing $T_{0}$ also contains $s_{0}$.

For the computation in practice, let $\bar{e}_{j}$ be defined by $e_{j} \equiv \bar{e}_{j}\left(\bmod p^{M}\right)$, as in $\S 6$, and set

$$
\bar{s}_{0}=\frac{1}{p} \sum_{j=1}^{J} \bar{e}_{j} \bar{T}_{0}^{j},
$$

where $\bar{T}_{0}$ is given by Proposition 10 .

Proposition 11. If $J$ is large enough and $\rho M \geq \gamma+e$, then

$$
s_{0} \equiv \bar{s}_{0} \quad\left(\bmod \pi^{\rho M-\gamma-e}\right) .
$$

The proof, which also implies an estimate for $J$, appears in $\S 12$ of the Supplements section.

\section{Starting the Newton Algorithm}

We are left with the question of how to start the Newton algorithm leading to an approximation of a zero $T_{0}$ of $f_{\theta}(T)$.

Suppose that $v_{p}\left(T_{0}\right)$ is known. In fact, we assume that we know the Newton polygon of $f_{\theta}(T)$, that is, the $p$-adic values of sufficiently many of its first coefficients $a_{0}, a_{1}, \ldots$ (these values were computed in Program A).

More precisely, we are going to find the (minimal) extension $E$ of $\mathbb{Q}_{p}$ containing $T_{0}$ and to determine $t_{0} \in \pi \mathscr{O}_{E}$ so that $f_{\theta}\left(t_{0}\right) \equiv 0\left(\bmod \pi^{2 \gamma+1}\right)$, where $\gamma=v_{\pi}\left(f^{\prime}\left(t_{0}\right)\right)=v_{\pi}\left(f^{\prime}\left(T_{0}\right)\right)$. Then, by Proposition 8, $t_{0}$ works as an initial approximation in the computation of $T_{0}$. In particular, $T_{0} \equiv t_{0}\left(\bmod \pi^{\gamma+1}\right)$, so that one can proceed in the way described in $\S 8$ (Proposition 10).

The above conditions for $t_{0}$ include the implicit assumption that the zero $T_{0}$ be simple. This was satisfied in all examples computed by us. We point out that Ferrero and Greenberg [4] have proved that $s_{0}=0$ is at most a simple 
zero of $L_{p}(s, \chi)$. From this it follows that $T_{0}=0$ never occurs as a multiple zero of $f_{\theta}(T)$. Whether the same holds true for nonzero $T_{0}$ is not known.

In the following, we set

$$
a_{j}=\sum_{k=0}^{\infty} a_{j k} p^{k} \quad \text { with } 0 \leq a_{j k}<p \quad(j=0,1, \ldots)
$$

and restrict ourselves to two basic types of Newton polygon met in the computations. Our discussion not only covers the majority of all examples but also presents the essential features of the required techniques in most, though not all, of the remaining cases. The treatment of these will be completed in Part II.

The first type consists of polygons determined by the conditions $v_{p}\left(a_{0}\right)=1$, $\lambda$ prime to $p$. Hence, there is but one (finite) nonzero slope, and this equals $-1 / \lambda$. For any zero $T_{0}$ one has $v_{p}\left(T_{0}\right)=1 / \lambda$, so that $E / \mathbb{Q}_{p}$ is fully ramified of degree $\lambda$. Set $q=(\lambda, p-1)$. Since the ramification is tame, we know (e.g., [5, Ch. II, §16]) that $E$ is one of the $q$ (nonconjugate) fields

$$
\mathbb{Q}_{p}(\pi), \quad \pi^{\lambda}=r p \quad \text { with } \quad r \equiv g^{b} \quad(\bmod p), \quad 0 \leq b \leq q-1,
$$

where $g$ is a fixed primitive root of $p$. We also have

$$
\gamma=v_{\pi}\left(f^{\prime}\left(T_{0}\right)\right)=v_{\pi}\left(\lambda a_{\lambda} T_{0}^{\lambda-1}\right)=\lambda-1 .
$$

To determine $t_{0}$, assume that $\lambda>1$, the case $\lambda=1$ being trivial $\left(t_{0}=0\right)$. Write

$$
T_{0} \equiv x_{1} \pi \quad\left(\bmod \pi^{2}\right), \quad 0<x_{1}<p .
$$

By Krasner's lemma, this fixes $E=\mathbb{Q}_{p}\left(T_{0}\right)$ uniquely (up to conjugates), since $v_{p}(\pi-\alpha \pi)=1 / \lambda$ whenever $\alpha^{\lambda}=1$. Let us make this result explicit as follows. From $f_{\theta}\left(T_{0}\right) \equiv 0\left(\bmod \pi^{\lambda+1}\right)$ we have that $a_{01}+a_{\lambda 0} r x_{1}^{\lambda} \equiv 0(\bmod p)$, or

$$
r x_{1}^{\lambda} \equiv-a_{01} / a_{\lambda 0} \quad(\bmod p) .
$$

This yields a unique solution for $r \bmod p$, and $q$ solutions for $x_{1} \bmod p$, corresponding to $q$ zeros $T_{0} \in E$ (note that there are $\lambda / q$ extensions conjugate to $E$ ).

If $\lambda>2$, we go on by setting

$$
T_{0} \equiv x_{1} \pi+\cdots+x_{\lambda-1} \pi^{\lambda-1} \quad\left(\bmod \pi^{\lambda}\right) .
$$

Observe that

$$
T_{0}^{\lambda} \equiv x_{1}^{\lambda} \pi^{\lambda}+c_{1} \pi^{\lambda+1}+\cdots+c_{\lambda-2} \pi^{2 \lambda-2} \quad\left(\bmod \pi^{2 \lambda-1}\right),
$$

where the coefficients $c_{j}$ are of the form $\lambda x_{1}^{\lambda-1} x_{j+1}$ plus terms depending only on $x_{1}, \ldots, x_{j}$. The congruence $f_{\theta}\left(T_{0}\right) \equiv 0\left(\bmod \pi^{2 \gamma+1}\right)$ is easily seen to be equivalent to

$$
A_{1}+A_{2} \pi+\cdots+A_{\lambda-2} \pi^{\lambda-3} \equiv 0 \quad\left(\bmod \pi^{\lambda-2}\right)
$$

with each $A_{j}=A_{j}\left(x_{1}, \ldots, x_{j+1}\right) \in \mathbb{Z}$, hence equivalent to $A_{1} \equiv \cdots \equiv A_{\lambda-2} \equiv 0$ $(\bmod p)$. Since

$$
A_{j} \equiv \lambda a_{\lambda 0} r x_{1}^{\lambda-1} x_{j+1}+A_{j}^{\prime}\left(x_{1}, \ldots, x_{j}\right)(\bmod p),
$$

where $A_{j}^{\prime}$ does not depend on $x_{j+1}$, we find that $x_{j+1} \bmod p$ becomes uniquely determined once $x_{1}$ is fixed $(j=1, \ldots, \lambda-2)$. As final result we get that $t_{0}=x_{1} \pi+\cdots+x_{\lambda-1} \pi^{\lambda-1}$ satisfies the desired conditions. 
The second type we consider is the case in which $v_{p}\left(a_{0}\right)=2$ and $\lambda=2$. Then, for both zeros $T_{0}=T_{0}^{\prime}$ and $T_{0}^{\prime \prime}$, say, $v_{p}\left(T_{0}\right)=1$, and so $E$ is one of the fields $\mathbb{Q}_{p}, \mathbb{Q}_{p}(\sqrt{g}), \mathbb{Q}_{p}(\sqrt{p}), \mathbb{Q}_{p}(\sqrt{g p})$. In any case, we may write

$$
T_{0} \equiv x p \quad\left(\bmod p^{3 / 2}\right) \quad \text { with } x=u+v \sqrt{g} \neq 0,
$$

where $0 \leq u<p, 0 \leq v<p$. Now the congruence $f_{\theta}\left(T_{0}\right) \equiv 0\left(\bmod p^{5 / 2}\right)$ becomes

$$
a_{02}+a_{11} x+a_{20} x^{2} \equiv 0 \quad(\bmod p),
$$

and this gives us two different solutions $x \in \mathbb{Z}$ or $x \in \mathbb{Z}[\sqrt{g}]$ according to whether the discriminant $D=a_{11}^{2}-4 a_{02} a_{20}$ is a quadratic residue or nonresidue $\bmod p$, respectively, and a double root $x \in \mathbb{Z}$ if $D \equiv 0(\bmod p)$.

In the former case $(D \not \equiv 0)$ we have by Krasner's lemma $E=\mathbb{Q}_{p}$ for $x \in \mathbb{Z}$, while $E=\mathbb{Q}_{p}(\sqrt{g})$ for $x \in \mathbb{Z}[\sqrt{g}]$. Moreover, since $a_{11}+2 a_{20} x \not \equiv 0(\bmod p)$, we see that $\gamma=v_{p}\left(f^{\prime}\left(T_{0}\right)\right)=1$. In this case, $f_{\theta}(x p) \equiv 0\left(\bmod p^{3}\right)$, which shows that $t_{0}=x p$ works as an initial approximation.

Suppose that $D \equiv 0(\bmod p)$; then $v_{p}\left(T_{0}^{\prime}-T_{0}^{\prime \prime}\right) \geq 3 / 2$. We will postpone the cases with $v_{p}\left(T_{0}^{\prime}-T_{0}^{\prime \prime}\right) \geq 2$ to Part II and let here this distance be $3 / 2$. This means that the zeros are of the form $T_{0} \equiv x p \pm y p \sqrt{r p}\left(\bmod p^{2}\right)$, where $r=1$ or $g$ and $0<x<p, 0<y<p$. In particular, $E=Q_{p}(\pi)$ with $\pi=\sqrt{r p}$. Using the congruence $a_{11}+2 a_{20} x \equiv 0(\bmod p)$, we find that

$$
f_{\theta}^{\prime}\left(T_{0}\right) \equiv \pm 2 a_{20} y p \pi \quad\left(\bmod \pi^{4}\right),
$$

and so $\gamma=3$. Hence, to obtain $r$ and $t_{0}$ we must consider the congruence $f_{\theta}\left(T_{0}\right) \equiv 0\left(\bmod \pi^{7}\right)$. This is equivalent to

$$
a_{20} r y^{2} \equiv-b-a_{03}-a_{12} x-a_{21} x^{2}-a_{30} x^{3}(\bmod p),
$$

where $b \equiv\left(a_{02}+a_{11} x+a_{20} x^{2}\right) / p(\bmod p)$. Upon solving for $r$ and $y$, we are done: take $t_{0}=x p+y p \pi$.

\section{NUMERICAL RESUltS}

A table including the main results from Program A appears in the Supplements section of this issue. This table gives a first approximation for $f_{\theta}(T)$, where $\theta$ runs through all the characters treated in this program (about 1150 in number).

A sample of the results from Program B is exhibited in Tables I-V of the Supplements. The present section contains a description of these tables together with some further examples.

Each item in the tables is headed by a triple $(p, \Delta, t)$ identifying the character $\theta=\theta_{m} \omega^{t}$. Here, $\Delta$ denotes the discriminant of $\mathbb{Q}(\sqrt{m})$, hence $\Delta=m$ or $4 m$ and $|\Delta|$ equals $d$, the conductor of $\theta_{m}$.

Of the two columns below the triple, the first (left) lists the first coefficients of $L_{p}(s, \theta)=\sum_{i=0}^{\infty} u_{i} s^{i}$ and the second (right) those of $f_{\theta}(T)=\sum_{j=0}^{\infty} a_{j} T^{j}$. For example, the first item of Table I (with $p=3$ and $\theta=\theta_{-47} \omega$ ) gives the 
following data about the coefficients:

$$
\begin{array}{ll}
u_{0}=0 & a_{0}=0 \\
u_{1}=0.0020 \cdots & a_{1}=0.0100 \cdots \\
u_{2}=0.0120 \cdots & a_{2}=1.1222 \cdots \\
u_{3}=0.0012 \cdots & a_{3}=1.002 \cdots \\
u_{4}=0.0010 \cdots & a_{4}=2.00 \cdots \\
u_{5}=0.0000 \cdots & a_{5}=1.1 \cdots \\
u_{6}=0.0001 \cdots & a_{6}=(1 .) \cdots
\end{array}
$$

All the nonzero $p$-adic numbers are written in the "decimal" form:

$$
\sum_{\nu=-a}^{b} x_{\nu} p^{\nu}=x_{-a} x_{-a+1} \cdots x_{0} \cdot x_{1} x_{2} \cdots x_{b} \quad\left(0 \leq x_{\nu}<p, a \geq 0, b \geq 0\right) .
$$

For $p=11$ the digit $x_{\nu}=10$ is denoted by $\alpha$.

The coefficient columns are followed by the pairs $T_{k}, s_{k}$, for $k=1, \ldots, \lambda$, where $T_{k}$ is a zero of $f_{\theta}(T)$ and $s_{k}=\log \left(1+T_{k}\right) / \log (1+d p)$. Observe that $v_{p}\left(s_{k}\right)=v_{p}\left(T_{k}\right)-1$ if $T_{k} \neq 0$ and $v_{p}\left(T_{k}\right)>1 /(p-1)$. When $s_{k}$ is not a zero of $L_{p}(s, \theta)$, it is denoted by $s_{k}^{*}$; this occurs only in Tables IV and V. In case $T_{k}$ generates a proper extension $E$ of $\mathbb{Q}_{p}$, it is preceded by a "standard" generator of $E$. If $E / \mathbb{Q}_{p}$ is ramified, this generator, a prime element of $\mathscr{O}_{E}$, is denoted by $\pi$ (or $\pi^{\prime}, \pi_{k}$ ), otherwise by $\xi$.

Each nonzero value of $u_{i}, a_{j}, T_{k}, s_{k}$ is an approximation. When we write 0 for a value of these numbers, it is exact. The approximations are truncated, according to the above propositions, to contain only correct digits. To save space, we in many cases list the coefficients $u_{i}$ and $a_{j}$ with an accuracy that is lower than actually computed. However, when tabulating $u_{i}$, we follow the principle that all the coefficients which are nonzero to the displayed accuracy are included (cf. (5.3) and (5.4)). Thus, in the above example,

$$
u_{i}=0.0000 \cdots \quad \text { for all } i \geq 7 \text {. }
$$

We now comment on the tables separately and offer some additional examples.

Table I provides examples in which $\lambda=2$ and $a_{0}=0$. This is an easy case: apart from the trivial zero $T_{1}=0, f_{\theta}(T)$ has a unique zero $T_{2} \in \mathbb{Q}_{p}$ and this can be computed by replacing $f_{\theta}(T)$ with $f_{\theta}(T) / T$ and choosing 0 as an initial approximation. In the examples of this type, it is not rare that $v_{p}\left(T_{2}\right)>1$, while the previously known examples (with $\lambda=1$ ) always are about zeros with $p$ ordinal 1. The maximum values we found for $v_{p}\left(T_{2}\right)$ occurred in the following cases:

EXAMPLE 1.

$$
\begin{aligned}
(3,-971,1): & T_{1}=0, \quad s_{1}=0, \\
T_{2} & =0.000011, \quad s_{2}=0.00021 .
\end{aligned}
$$

EXAMPLE 2.

$$
\begin{aligned}
(3,-2351,1): & T_{1}=0, \quad s_{1}=0, \\
T_{2} & =0.0000021, \quad s_{2}=0.000012 .
\end{aligned}
$$

In Table II we have $\lambda=2$ and $a_{0} \neq 0$. Here we give only examples with $v_{p}\left(T_{k}\right)=1 / 2$ or 1 ; the cases with higher $v_{p}\left(T_{k}\right)$ will be discussed in Part II. 
Note that if $p=3$ and $v_{3}\left(T_{k}\right)=1 / 2, s_{k}$ is not a zero of $L_{3}(s, \theta)$. These cases are postponed to Part II as well.

The next example, being of the type of Table II, corrects some erroneous data in [13].

EXAMPLE 3.

$$
\begin{aligned}
(5,109,0): T_{1,2} & =0.4122 \pm 2.1134 \sqrt{2 \cdot 5} \\
s_{1,2} & =0.0322 \pm 33.211 \sqrt{2 \cdot 5}
\end{aligned}
$$

(With the definition of $f_{\theta}(T)$ used in [13] one has $T_{1,2}=0.4434 \pm$ 2.3244 $\sqrt{2 \cdot 5}$.)

The examples in Table IIa, with $\lambda=3$ and $a_{0}=0$, are analogous to those in Table II: exclude the trivial zero $T_{1}=0$ and consider the power series $f_{\theta}(T) / T$.

Table III is about the case $\lambda=3, a_{0} \neq 0$. Except for one example, the zeros lie in ramified cubic extensions. The number of such extensions (not counting conjugates) is one for $p=5$, and three for $p=7$. For $p=3$, the ramification is wild. This case will be studied in Part II, but below we give just one example completing a discussion begun in [13].

EXAMPLE 4.

$$
\begin{aligned}
(3,281,0): & \pi_{k} \text { zero of } X^{3}-6 X-3 \quad(k=1,2,3), \\
& T_{k} \equiv \pi_{k}+\pi_{k}^{3}\left(\bmod \pi_{k}^{5}\right), \\
& \text { no zero } s_{k} \text { corresponding to } T_{k} .
\end{aligned}
$$

The next example, still about $p=3$, falls into the same category $(\lambda=3$, $\left.a_{0} \neq 0\right)$ but is of another kind: $f_{\theta}(T)$ has a linear factor. This example was also computed in [13] (with some errors in the last digits).

EXAMPLE 5.

$$
\begin{aligned}
(3,733,0): & T_{1}=0.12001, \quad s_{1}=1.1220, \\
& \pi=\sqrt{-3}, \\
& T_{2,3}=0.20120 \pm 1.12000 \pi \\
& s_{2,3}^{*}=1.2022 \pm 2.1010 \pi
\end{aligned}
$$

( $s_{2}^{*}$ and $s_{3}^{*}$ are zeros of $L_{3}\left(s, \theta \psi_{1}\right)$; see Part II).

Table IIIa is again a companion to the preceding table, with $\lambda=4$ and $a_{0}=0$.

Table IV lists examples in the case $\lambda=4, a_{0} \neq 0$. In all examples, $v_{p}\left(T_{k}\right)=$ $1 / 4$. Thus, we know about $s_{k}$ that it is a zero of $L_{p}(s, \theta)$ for $p=7$ but no zero of any $L_{p}\left(s, \theta \psi_{n}\right)$ for $p=3$. For $p=5$, the answer depends on $\mathbb{Q}_{5}(\pi)$; in our examples $\mathbb{Q}_{5}(\pi)$ never happens to be $\mathbb{Q}_{5}(\sqrt{-5})=\mathbb{Q}_{5}\left(\zeta_{5}\right)$, and so there is no $s_{k}$ corresponding to $T_{k}$ (see Proposition 5).

Finally, Table V contains three examples with $\lambda=5$ and one with $\lambda=6$. (In the last case, $\lambda$ was not computed in [3].) Again, most of the zeros $T_{k}$ lie in quartic extensions, and the $s_{k}$ obtained from these are no zeros of $L_{p}(s, \theta)$, not even zeros of $L_{p}\left(s, \theta \psi_{n}\right)$.

The previously computed tables $[3,8]$ list 12 further examples with $\lambda \geq 6$. We computed $v_{p}\left(T_{k}\right)$ for all zeros $T_{k}$ in these examples. There are three cases (with $p=3$ ) in which some of the zeros lie in wildly ramified sextic extensions, 
and we will return to them in Part II. In the remaining cases one can determine the extension $E=\mathbb{Q}_{p}(\pi)$, up to conjugates, from $v_{p}\left(T_{k}\right)$; they are as follows:

\begin{tabular}{|c|r|c|c|c|c|}
\hline$p$ & $\Delta$ & $t$ & $\lambda$ & $\pi$ & Remarks \\
\hline 3 & $-239,-4088,-1427,-17252,-36140$ & 1 & 6 & $\sqrt[5]{3}$ & $T_{1}=0$ \\
3 & -21592 & 1 & 7 & $\sqrt[7]{3}$ & - \\
3 & $-11156,-30584$ & 1 & 8 & $\sqrt[7]{3}$ & $T_{1}=0$ \\
7 & -1371 & 1 & 7 & $\sqrt[5]{7}$ & $T_{1}=0, T_{2} \in \mathbb{Q}_{7}$ \\
\hline
\end{tabular}

On the basis of the numerical data computed so far it seems natural to expect that the zeros $T_{i}$ and $s_{i}$ are distributed randomly as regards their $p$-adic value (within the prescribed limits) and their inclusion in various extensions of $\mathbb{Q}_{p}$. Also the $p$-adic expansions of the zeros fail to show any regularity.

\section{BIBLIOGRAPHY}

1. Z. I. Borevich and I. R. Shafarevich, Number theory, Academic Press, New York and London, 1966.

2. N. Childress and R. Gold, Zeros of p-adic L-functions, Acta Arith. 48 (1987), 63-71.

3. R. Ernvall and T. Metsänkylä, A method for computing the Iwasawa $\lambda$-invariant, Math. Comp. 49 (1987), 281-294.

4. B. Ferrero and R. Greenberg, On the behaviour of p-adic L-functions at $s=0$, Invent. Math. 50 (1978), 91-102.

5. H. Hasse, Zahlentheorie, 2. Aufl., Akademie-Verlag, Berlin, 1963.

6. E. L. Ince, Cycles of reduced ideals in quadratic fields, Math. Tables IV, British Assoc. Advancement Sci., London, 1934.

7. K. Iwasawa and C. Sims, Computation of invariants in the theory of cyclotomic fields, J. Math. Soc. Japan 18 (1966), 86-96.

8. S. Kobayashi, Calcul approché de la série d'Iwasawa pour les corps quadratiques $(p=3)$, Theorie des Nombres, 1981-82 and 1982-83, Exp. No. 4, 68 pp., Publ. Math. Fac. Sci. Besançon, Univ. Franche-Comté, Besançon, 1983.

9. T. Kubota and H. W. Leopoldt, Eine p-adische Theorie der Zetawerte, I: Einführung der p-adischen Dirichletschen L-Funktionen, J. Reine Angew. Math. 214/215 (1964), 328-339.

10. K. Lamprecht and H. G. Zimmer, p-adic algorithms and the computation of zeros of p-adic L-functions, EUROCAL '85, vol. 2 (Linz 1985), Lecture Notes in Comput. Sci., vol. 204, Springer, Berlin and New York, 1985, pp. 491-502.

11. R. F. Sunseri, Zeros of p-adic L-functions and densities relating to Bernoulli numbers, Ph.D. thesis, Univ. of Illinois at Urbana-Champaign, 1980.

12. S. S. Wagstaff, Jr., Zeros of p-adic L-functions, Math. Comp. 29 (1975), 1138-1143.

13. __ Zeros of p-adic L-functions, II, Number Theory Related to Fermat's Last Theorem (Cambridge, Mass., 1981), Progr. Math., vol. 26, Birkhäuser, Boston, Mass., 1982, pp. 297-308.

14. L. C. Washington, Introduction to cyclotomic fields, Springer, Berlin and New York, 1982.

15. __ Zeroes of p-adic L-functions, Séminaire de Théorie des Nombres, Paris 1980-81 (Paris, 1980/81), Progr. Math., vol. 22, Birkhäuser, Boston, Mass., 1982, pp. 337-357.

Department of Mathematics, University of Turku, SF-20500 Turku, Finland

E-mail address, R. Ernvall: rernvall@kontu.utu.fi

E-mail address, T. Metsänkylä: taumets@kontu.utu.fi 


\title{
Supplement to
}

\section{COMPUTATION OF THE ZEROS OF $p$-ADIC $L$-FUNCTIONS}

\author{
R. ERNVALL AND T. METSÄNKYLÄ
}

Here we present two supplementary sections, one ( $\$ 11)$ providing further details of the computations and another $(\S 12)$ giving the proofs of some propositions. These are followed by the numerical tables from Programs A and B as announced in the main text.

\section{Detalls of the computations}

As mentioned before, our computation of $L_{p}(s, \theta)=\sum_{i=0}^{\infty} u_{i} s^{i}$, i.e., the approximation of $u_{i}$ by $\bar{u}_{i} \bmod p^{M}$, was based on the expression given in (5.1). To study the structure of this expression, we write it in the form

$$
L_{p}(s, \theta)=-\frac{1}{d p} \sum_{\substack{a=1 \\ p \nmid a}}^{d p} \theta(a)\left(S_{1}(a)+S_{2}(a)+S_{3}(a)\right)
$$

where

$$
\begin{gathered}
S_{1}(a)=\sum_{j=1}^{\infty} \frac{1}{1-s}\left(\begin{array}{c}
1-s \\
j
\end{array}\right) \tau_{1}(a)^{j} \quad\left(\tau_{1}(a)=\langle a\rangle-1\right), \\
S_{2}(a)=\sum_{j=1}^{\infty} \frac{1}{1-s}\left(\begin{array}{c}
1-s \\
j
\end{array}\right) B_{j} \tau_{2}(a)^{j} \quad\left(\tau_{2}(a)=d p / a\right), \\
S_{3}(a)=(1-s) S_{1}(a) S_{2}(a)
\end{gathered}
$$

(note that the sum of $\theta(a)$ with $a=1, \ldots, d p, p \nmid a$, vanishes). Setting, moreover,

$$
\frac{1}{1-s}\left(\begin{array}{c}
1-s \\
j
\end{array}\right)=\sum_{i=0}^{j-1} \frac{c_{j i}}{j !} s^{i}
$$

with $c_{j i} \in \mathbf{Z}$, we have

$$
S_{\nu}(a)=\sum_{i=0}^{\infty} R_{\nu i} s^{i}, \quad R_{\nu i}=\sum_{j=i+1}^{\infty} \frac{c_{j i}}{j !} b_{\nu j} \tau_{\nu}(a)^{j} \quad(\nu=1,2),
$$

where $b_{1 j}=1$ and $b_{2 j}=B_{j}$. Note that $v_{p}\left(\tau_{\nu}(a)\right) \geq 1$.

From this it is seen that the principal task is the calculation of (rational) numbers $\bar{R}_{\nu i}$ satisfying

$$
R_{\nu i} \equiv \bar{R}_{\nu i} \quad\left(\bmod p^{M+1}\right) .
$$

These numbers are computed from the series of $R_{\nu i}$ by way of truncations of two kinds: the truncation of the series itself and that of the p-adic expansions of $c_{j i}, j !, b_{\nu j}$, and $\tau_{\nu}(a)$. Let $\eta_{1}$ and $\eta_{2}$ be parameters such that the series for $R_{\nu i}$ are computed up to $j=\eta_{1}$ and the above $p$-adic numbers are computed mod $p^{\eta_{2}}$. An optimal choice appears to be $\eta_{1}=\eta_{2}-1=\eta$, say. Between $M$ and $\eta$ there is the following relationship, proved in the next section. 

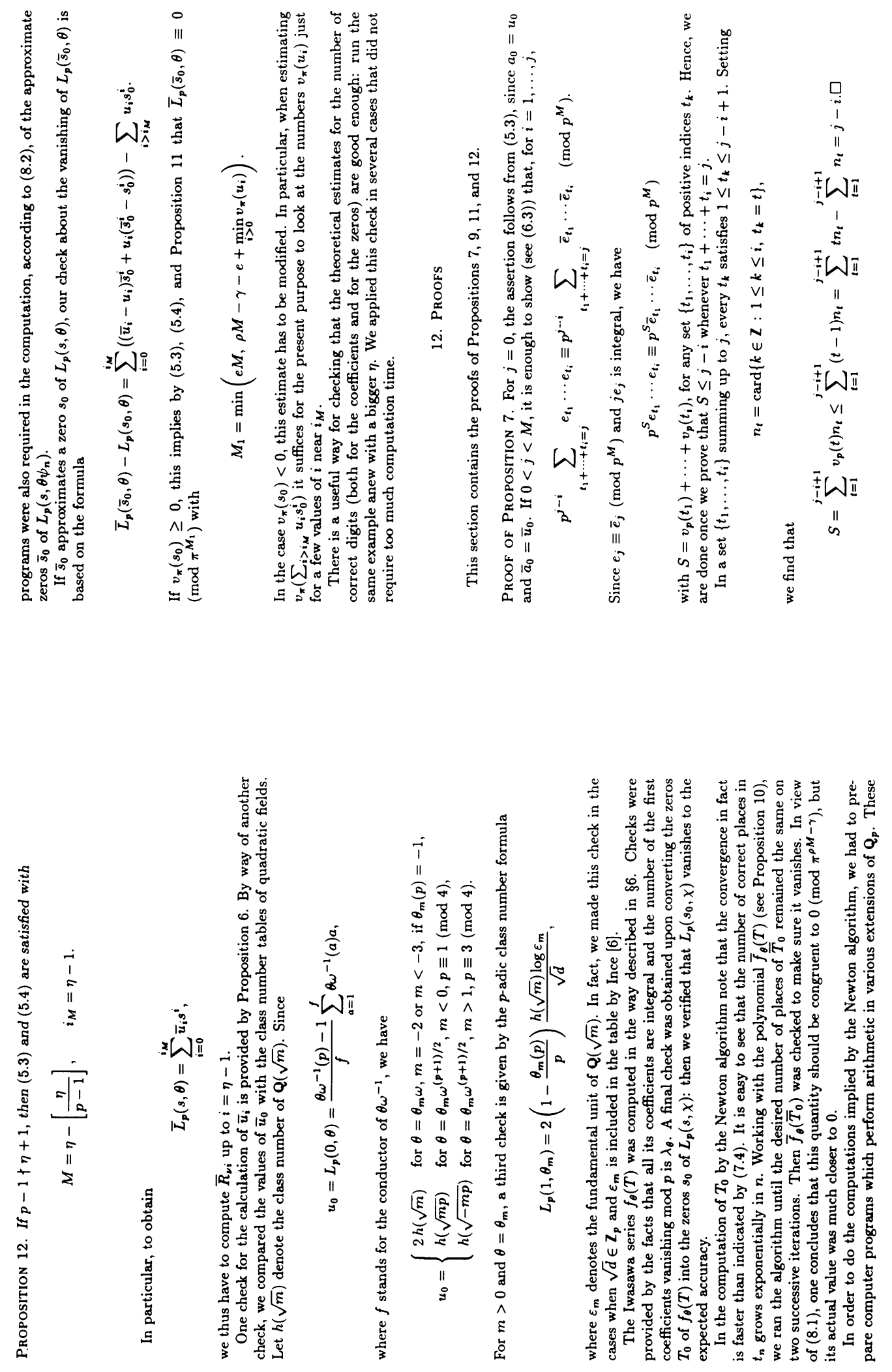

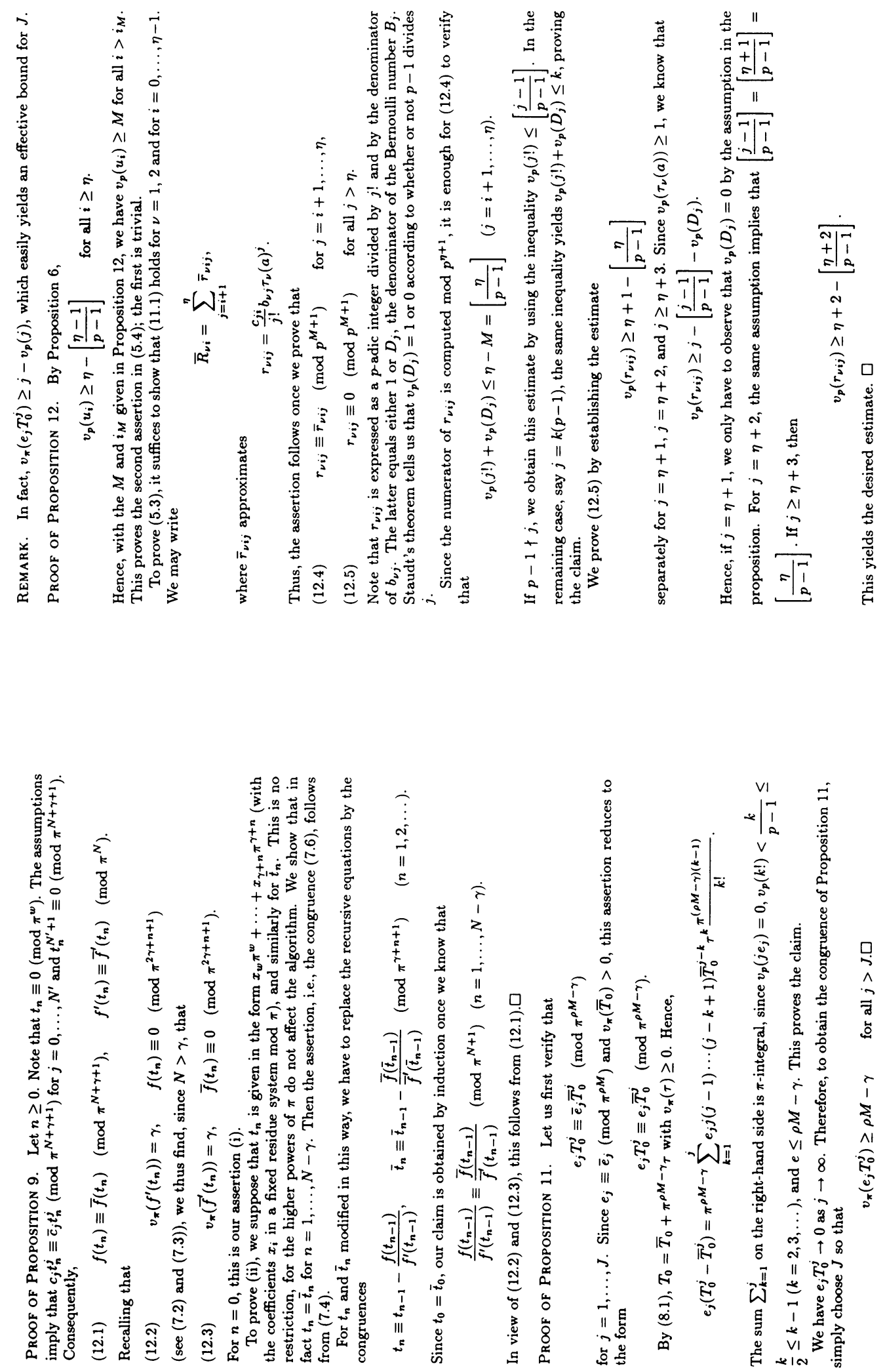


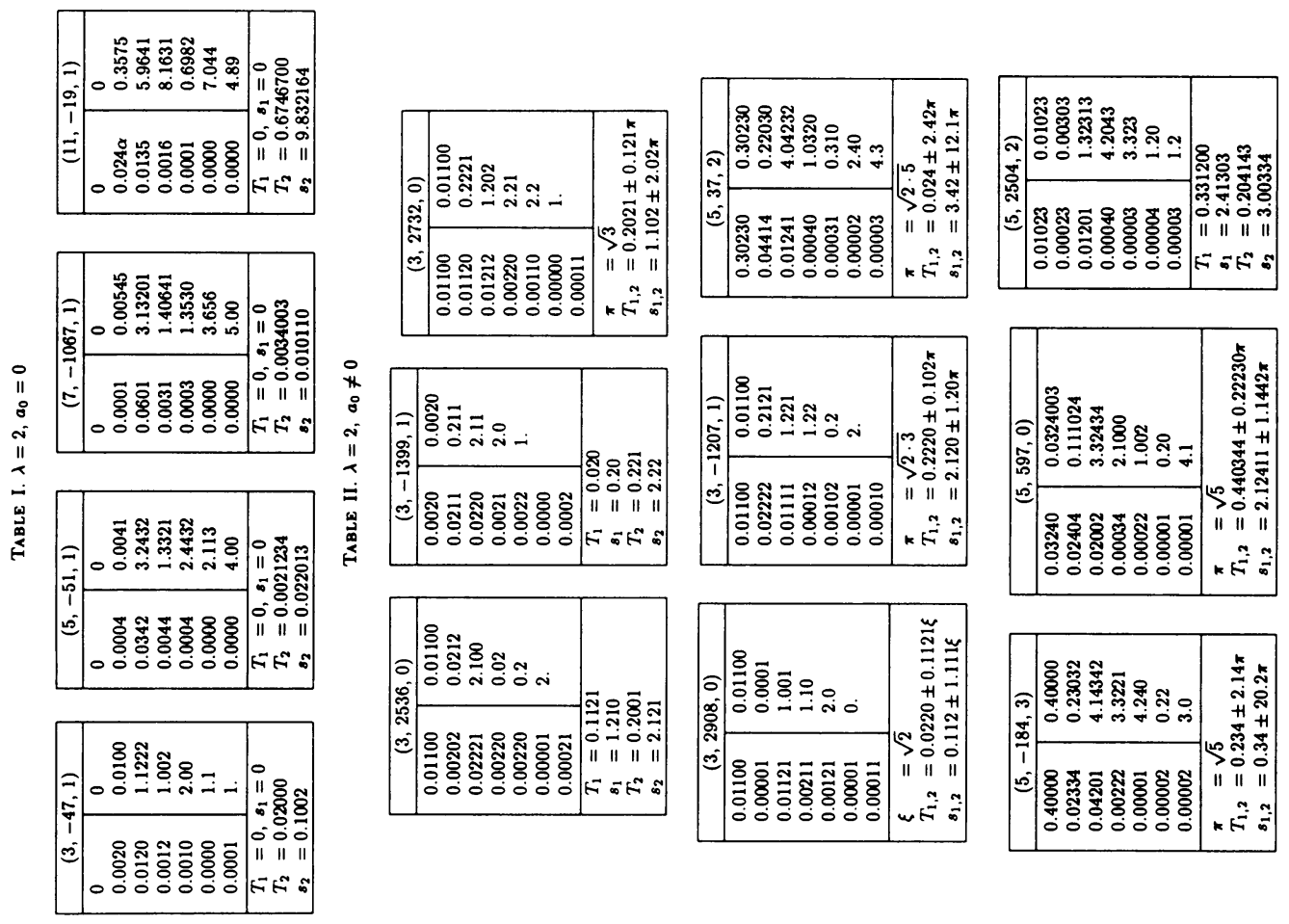

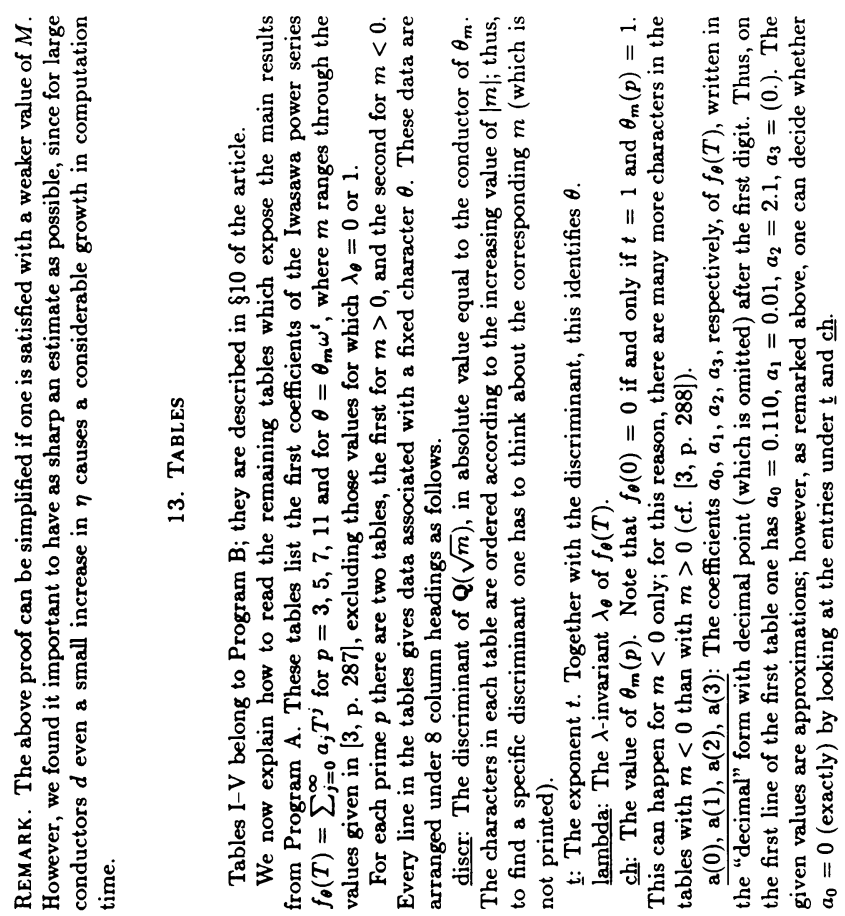



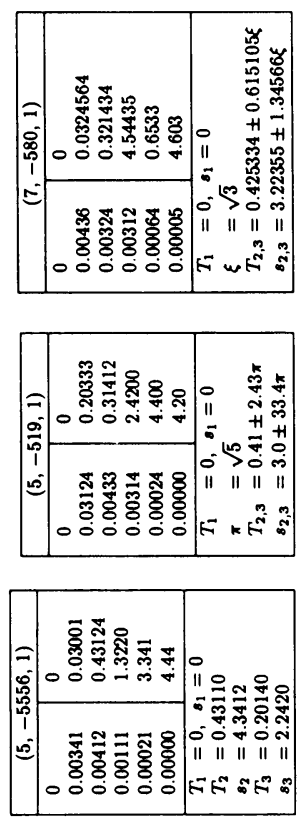
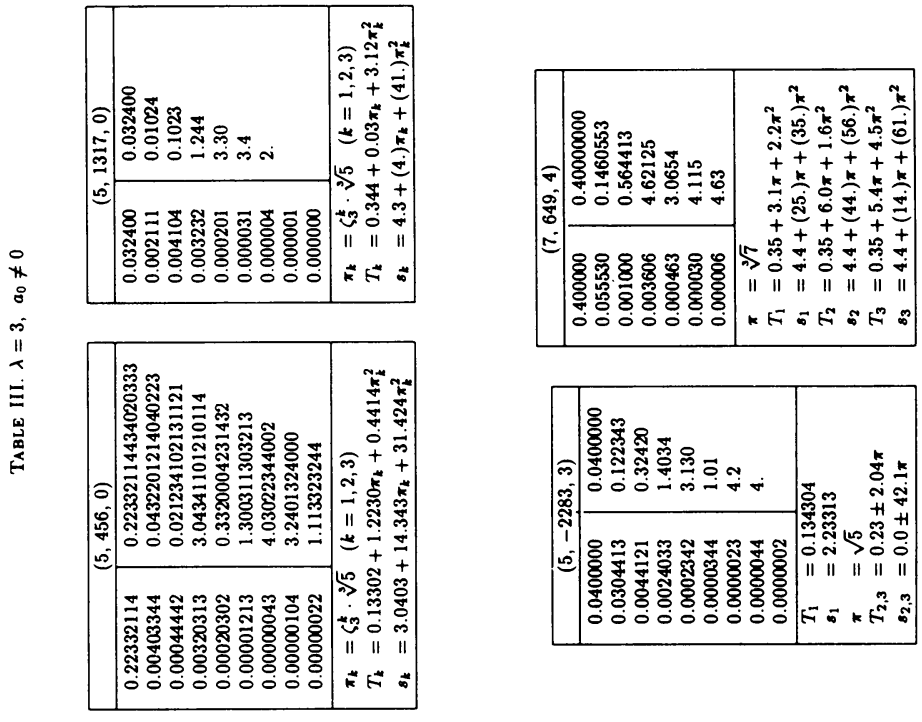
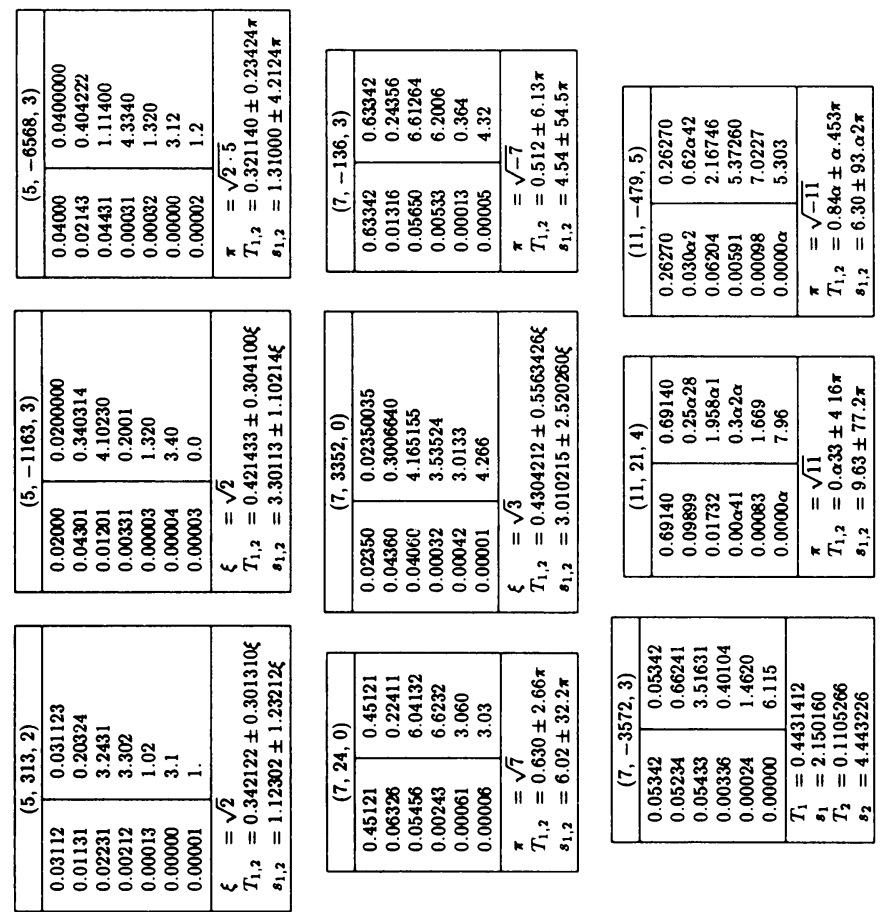
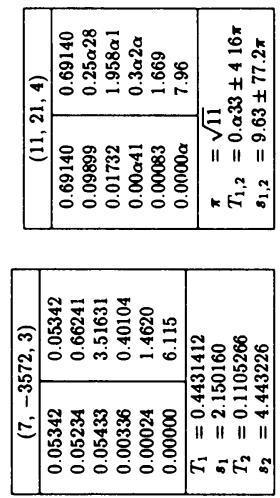
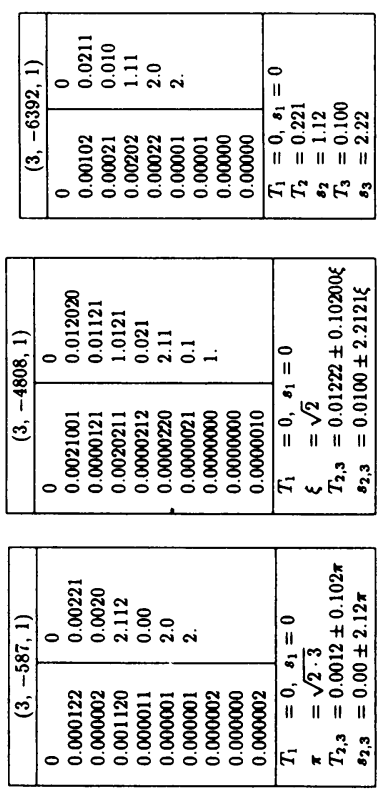

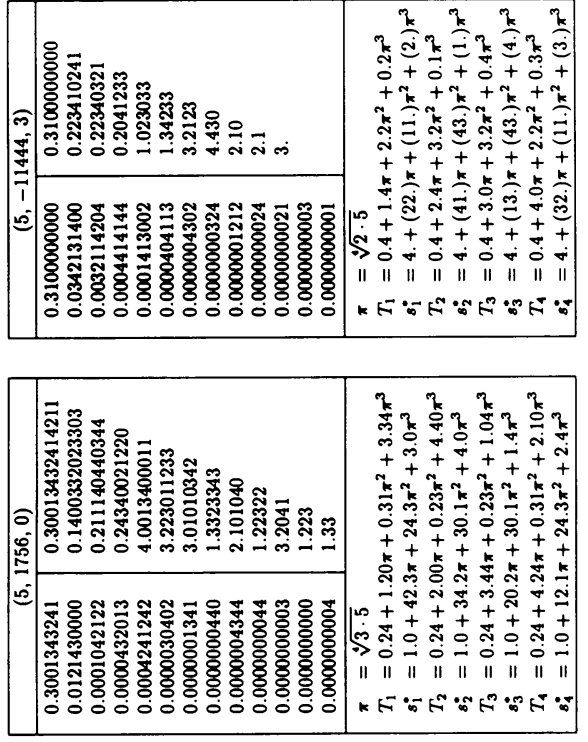
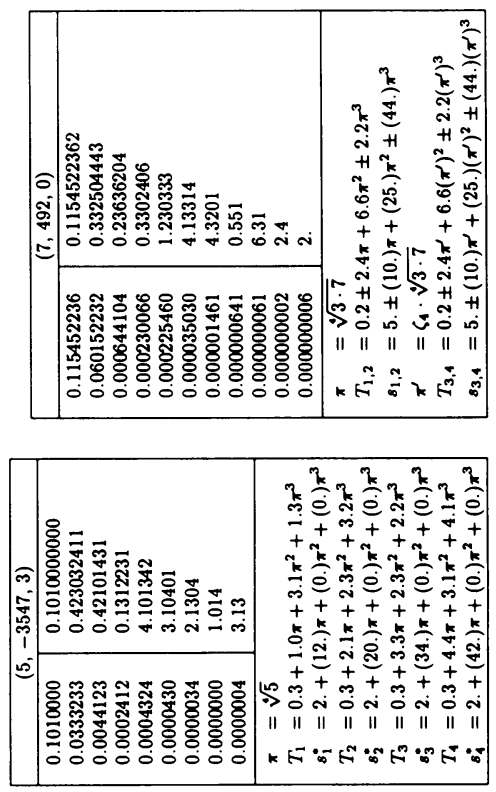
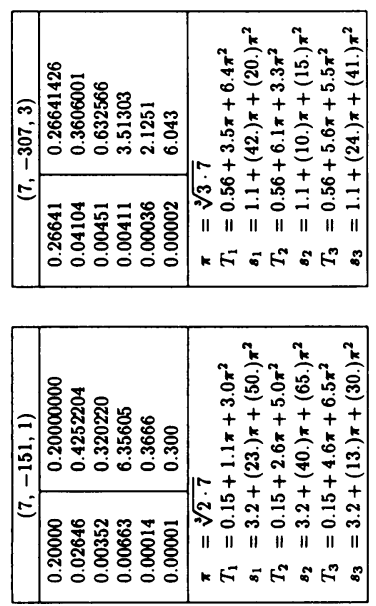
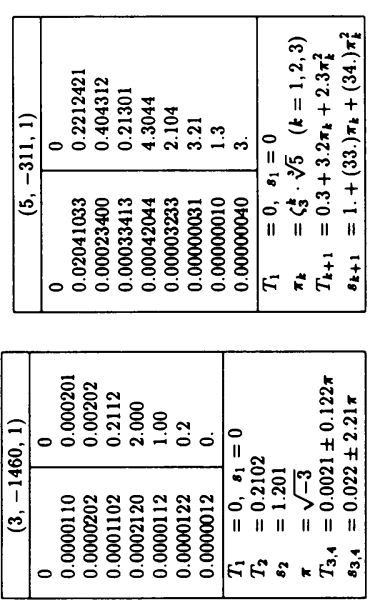

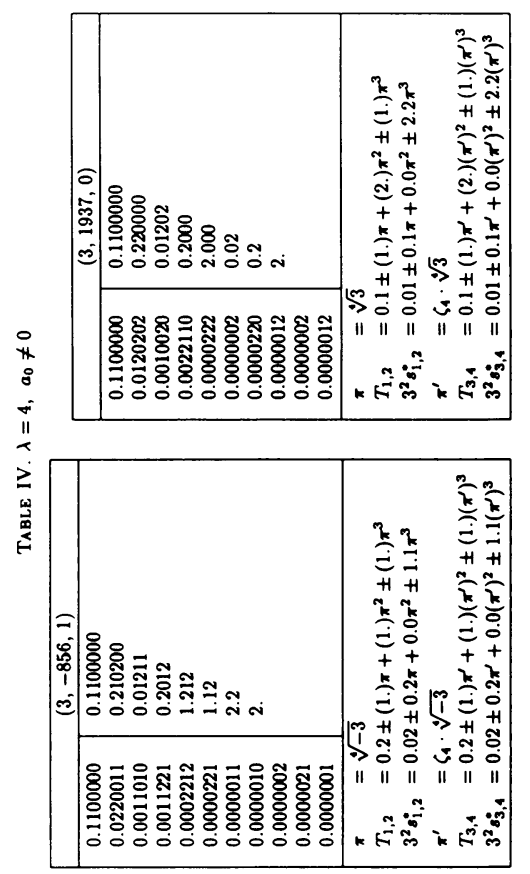



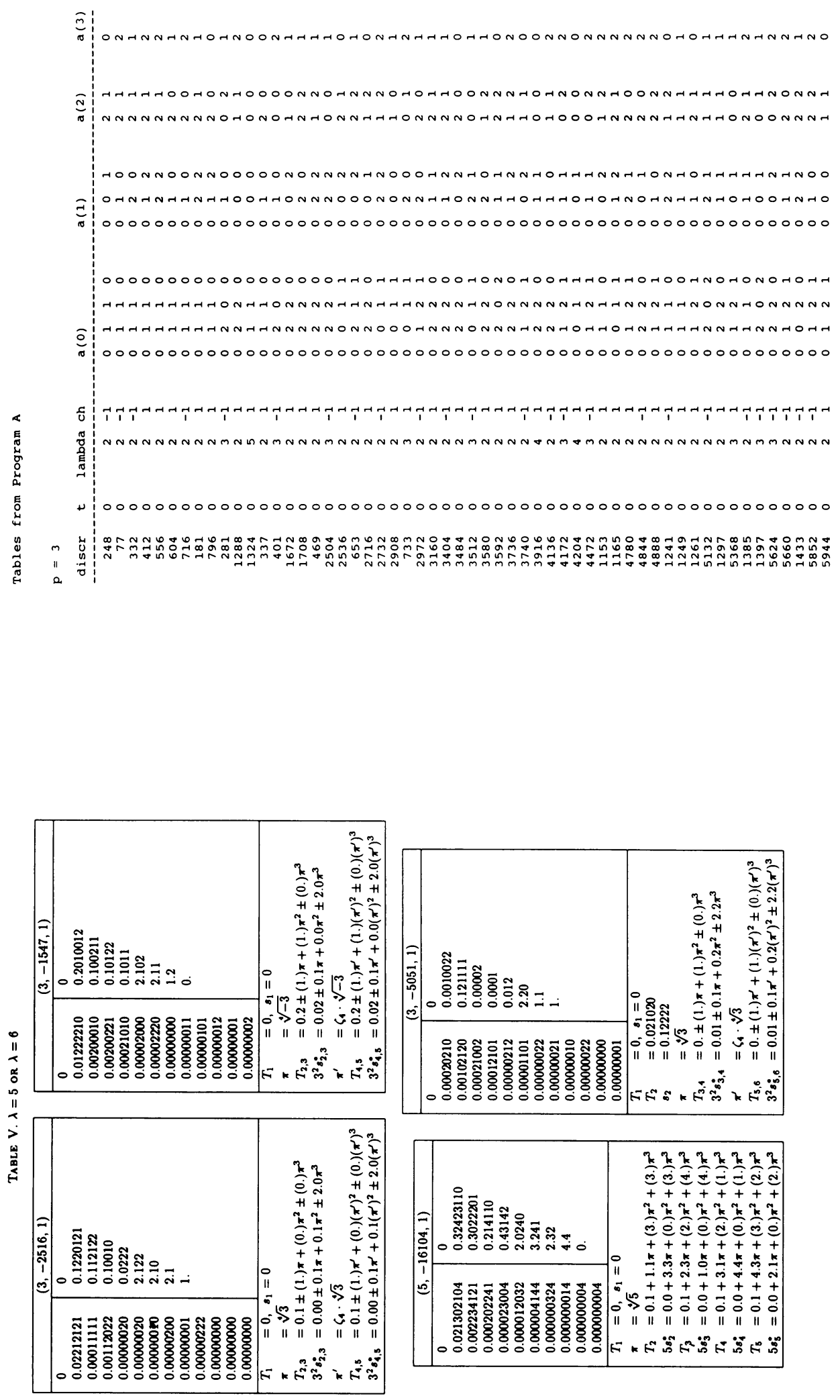


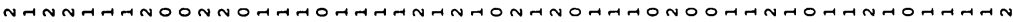

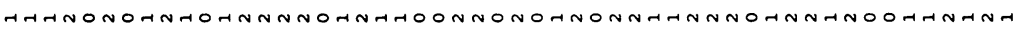

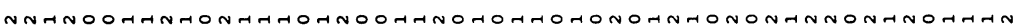

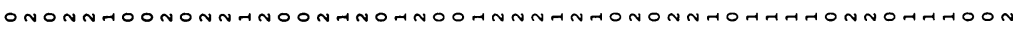

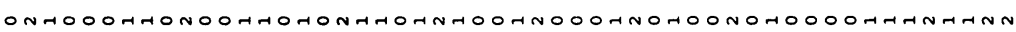
0000000000000000000000000000000000000000000000000000

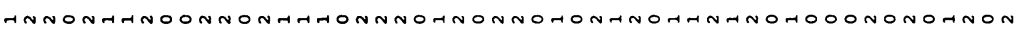
hon ON ONONNHTHONOOOHHONOOONHNHNNNHANHONHHOHONONNNNNNNOOHO 0000000000000000000000000000000000000000000000000000

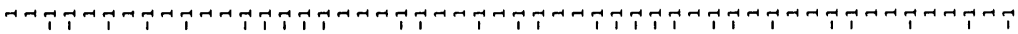

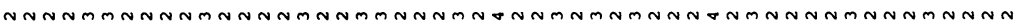
0000000000000000000000000000000000000000000000000000

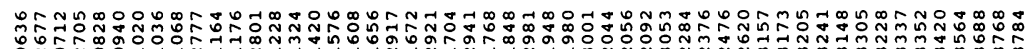

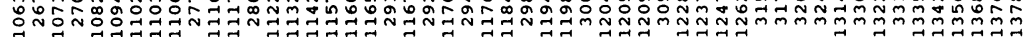

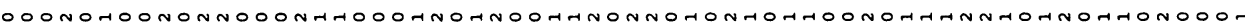

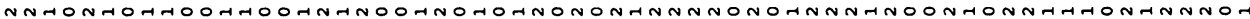

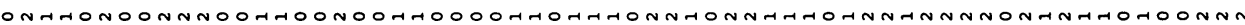

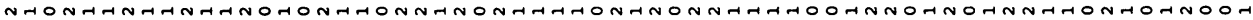

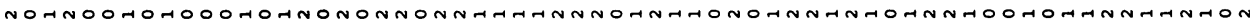
0000000000000000000000000000000000000000000000000000000000000000

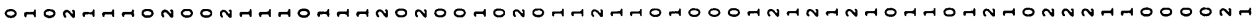

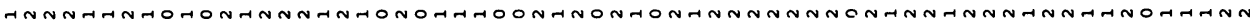

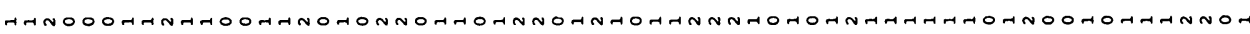
0000000000000000000000000000000000000000000000000000000000000000

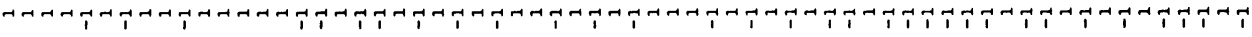

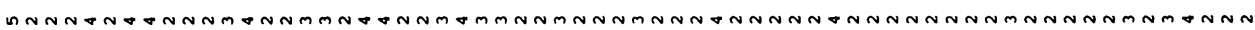




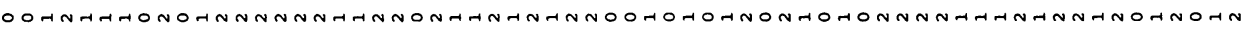

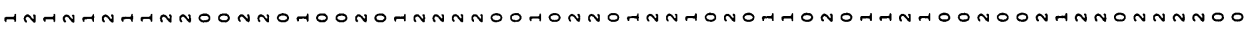

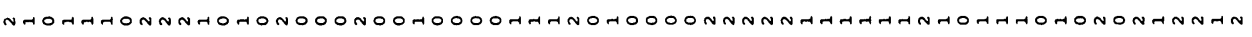

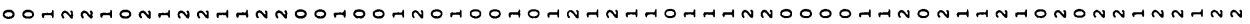

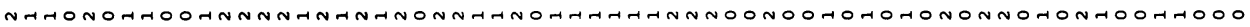
0000000000000000000000000000000000000000000000000000000000000000

$000000000400000000040000000000000000 n 0000004000 n 0040040000000470$

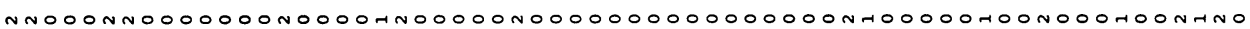

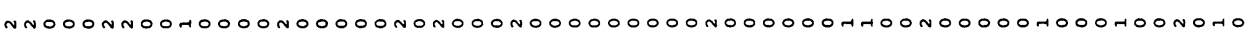
0000000000000000000000000000000000000000000000000000000000000000

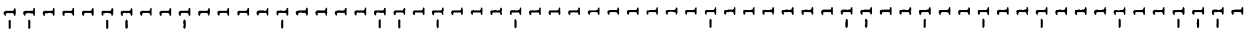
NNMNNNMNNNNMNMNMMMNMMNMMMMNNNNMNGMOMNNNNNNNNNNNNNMNNNMNMNMNNNNNN

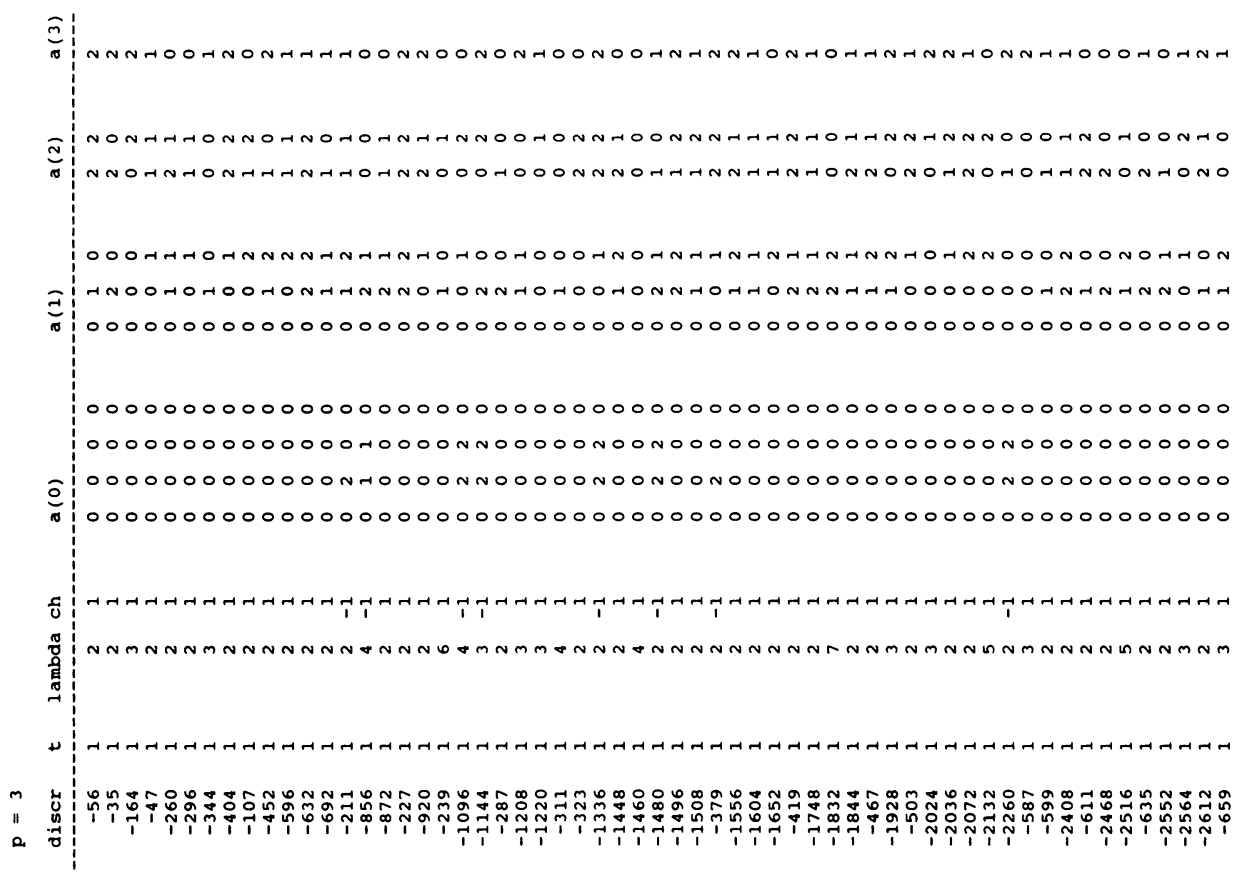




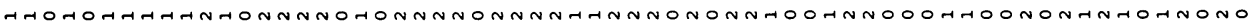

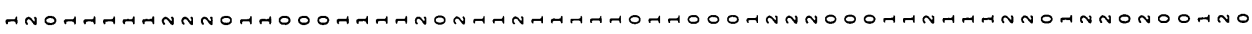

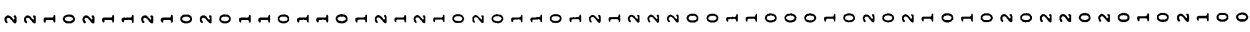

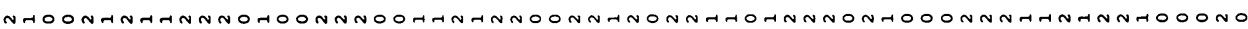

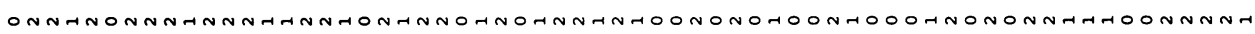
0000000000000000000000000000000000000000000000000000000000000000

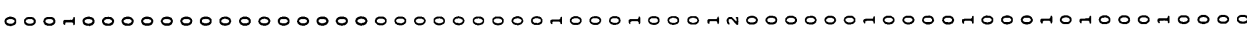

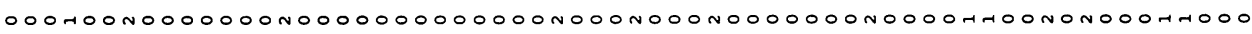

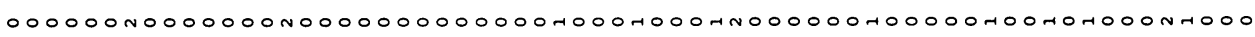
0000000000000000000000000000000000000000000000000000000000000000

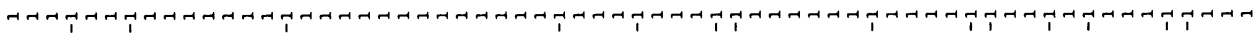

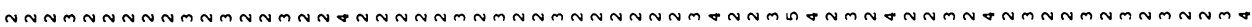

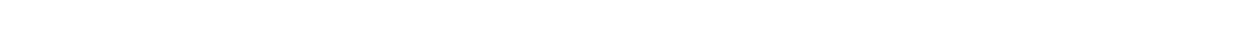

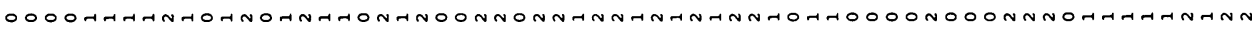

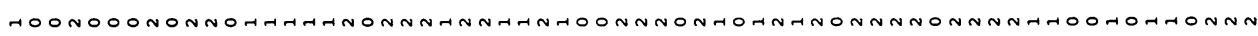
0000000000000000000000000000000000000000000000000000000000000000

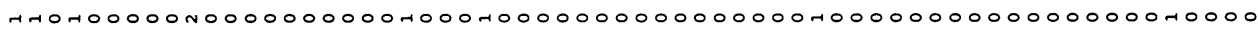

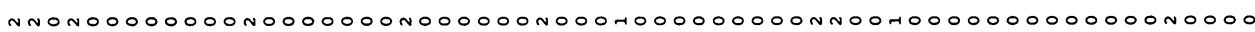

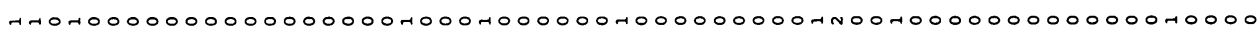
0000000000000000000000000000000000000000000000000000000000000000

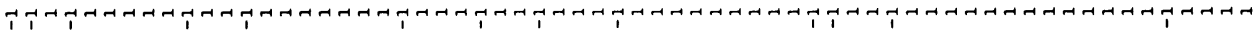

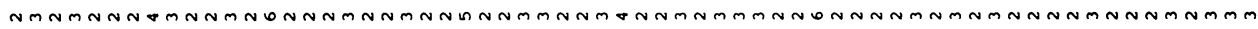




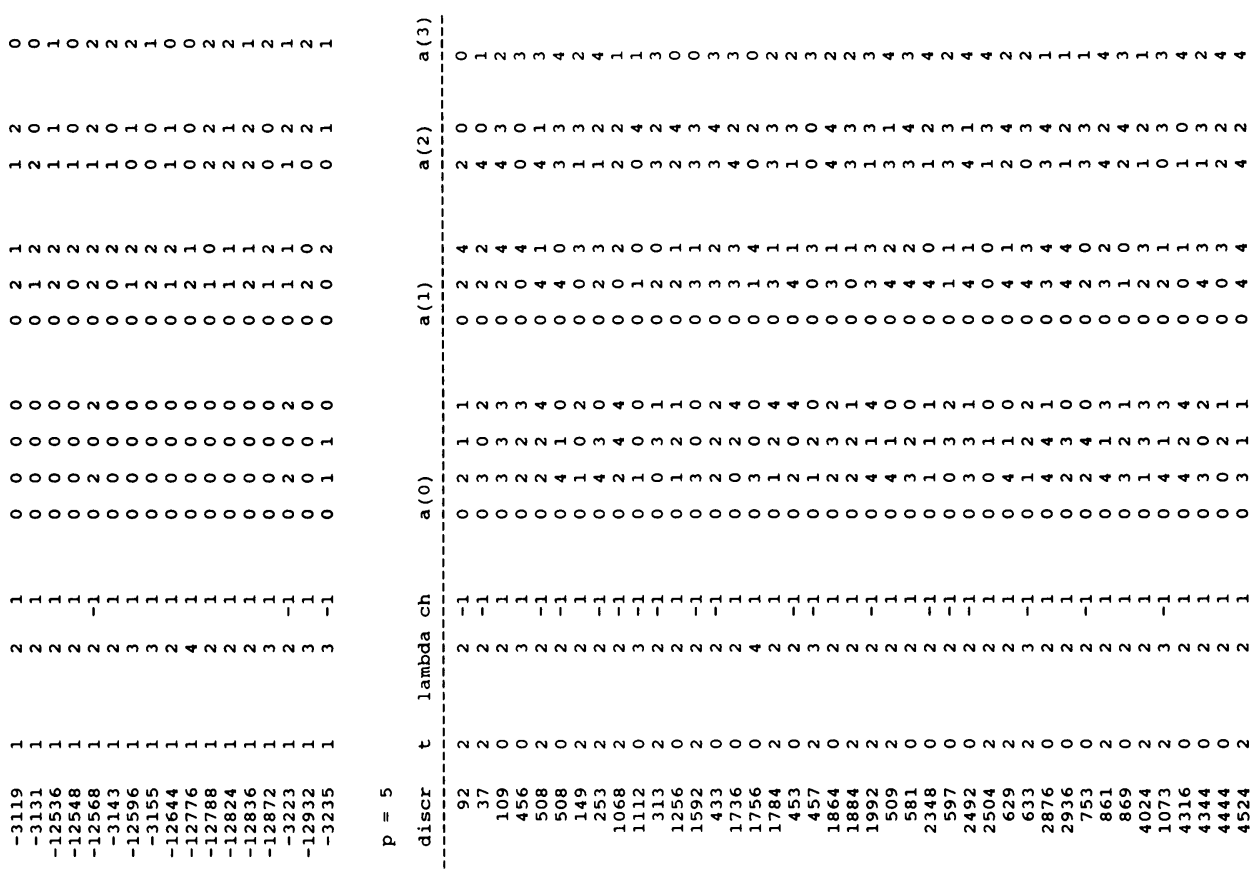

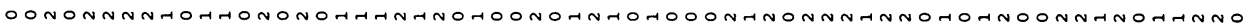

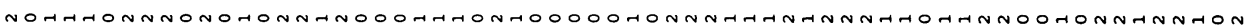

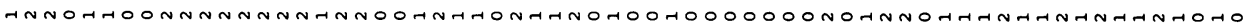

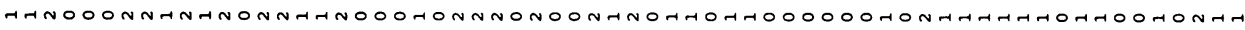

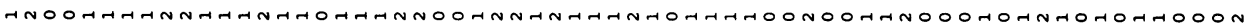
0000000000000000000000000000000000000000000000000000000000000000

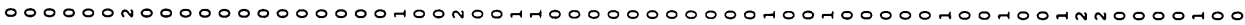

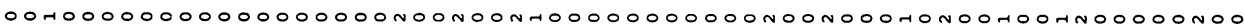

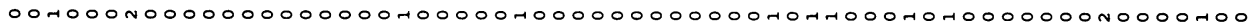
0000000000000000000000000000000000000000000000000000000000000000

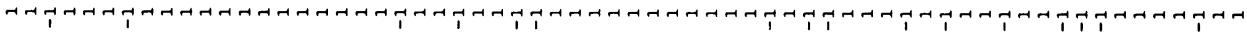

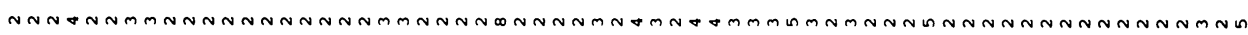




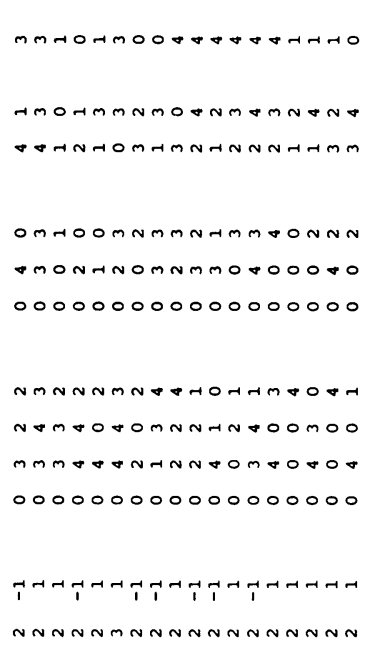

oo ononnnnnNoOnoon

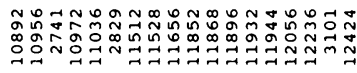

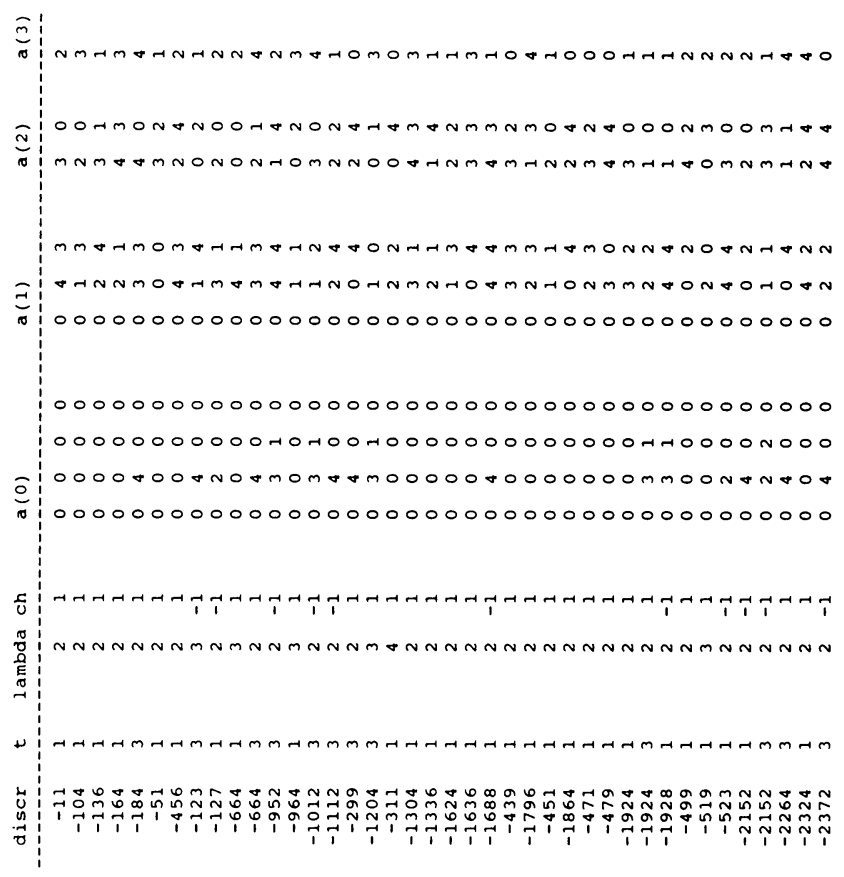

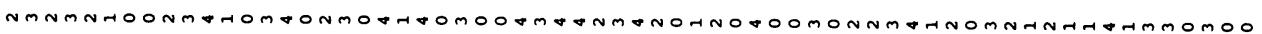

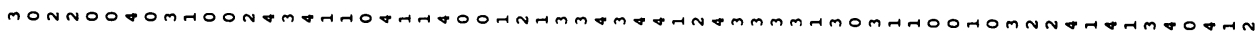
0000000000000000000000000000000000000000000000000000000000000000

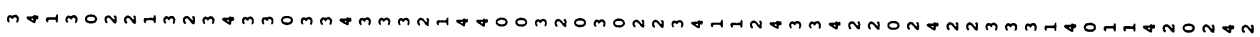

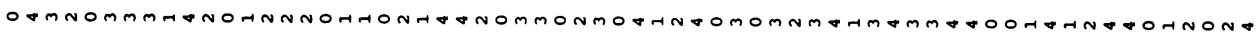

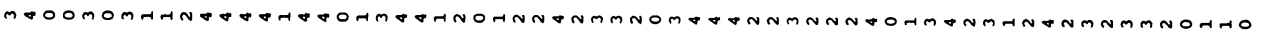
0000000000000000000000000000000000000000000000000000000000000000

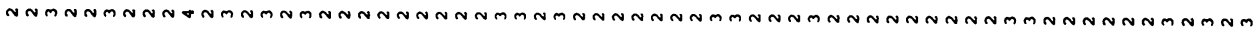




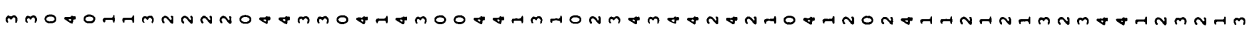

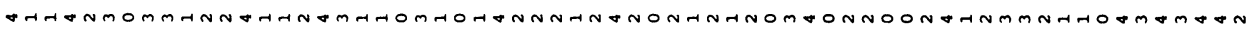

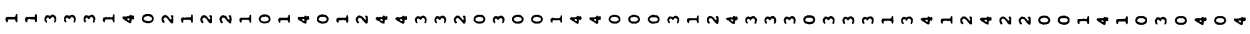

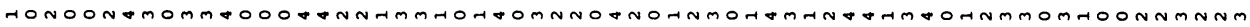

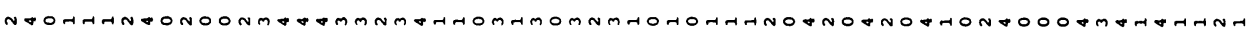
0000000000000000000000000000000000000000000000000000000000000000

0000000000000000000000000000000000000000000000000000000000000000

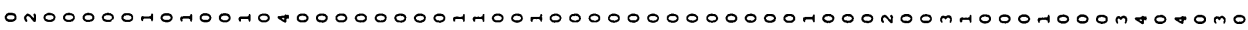

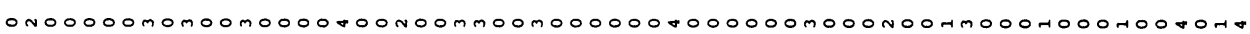
0000000000000000000000000000000000000000000000000000000000000000

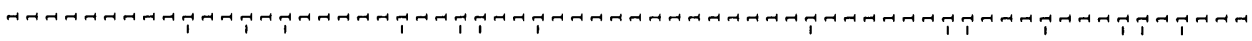

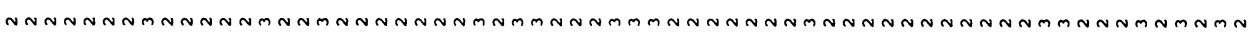

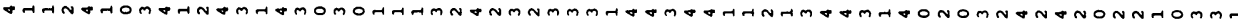

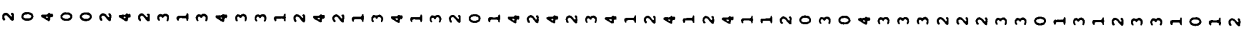
0000000000000000000000000000000000000000000000000000000000000000

0000000000000000000000000000000000000000000000000000000000000000 $0000000000000000004000000000 m 00070 n 0000040 N 0000 m 004 N 0000404 N 0000$

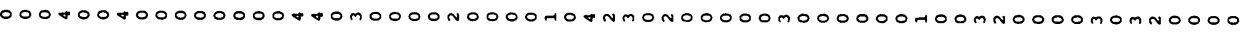
0000000000000000000000000000000000000000000000000000000000000000

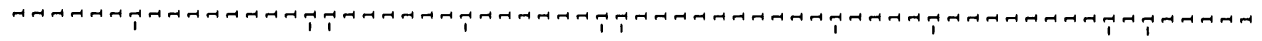

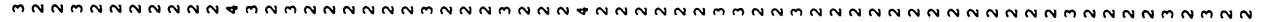




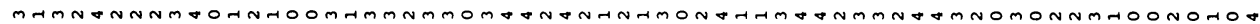

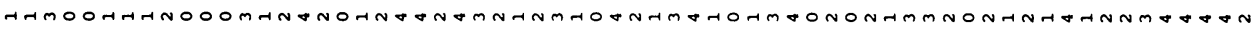

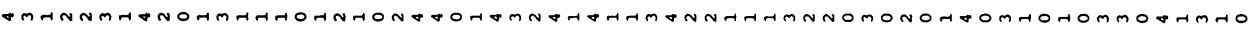

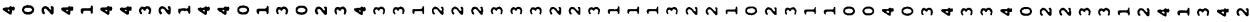

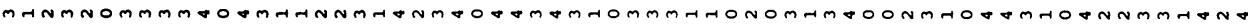
0000000000000000000000000000000000000000000000000000000000000000

$000007000000000000000007000070-1007000700000000000007000000000000$

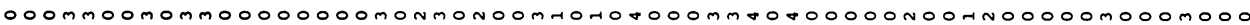

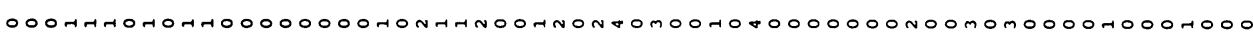
0000000000000000000000000000000000000000000000000000000000000000

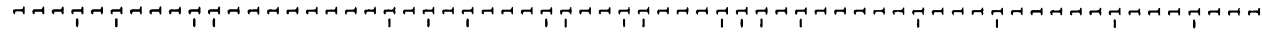

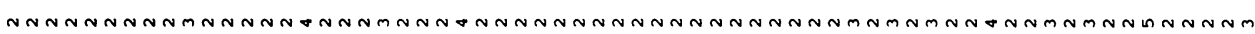

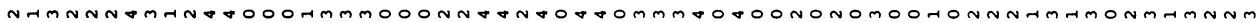

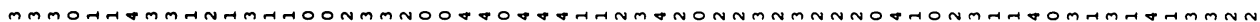
0000000000000000000000000000000000000000000000000000000000000000

$0000000000000000000000000-00000000000000000000000000000000700000$

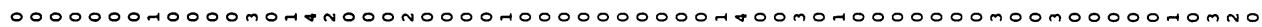

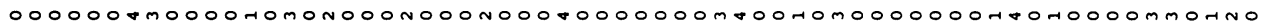
0000000000000000000000000000000000000000000000000000000000000000

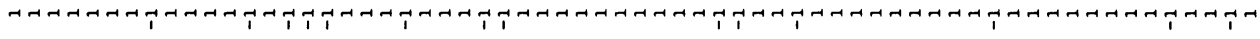

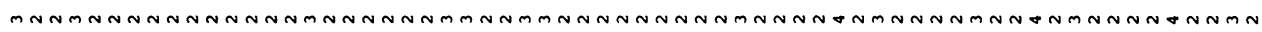



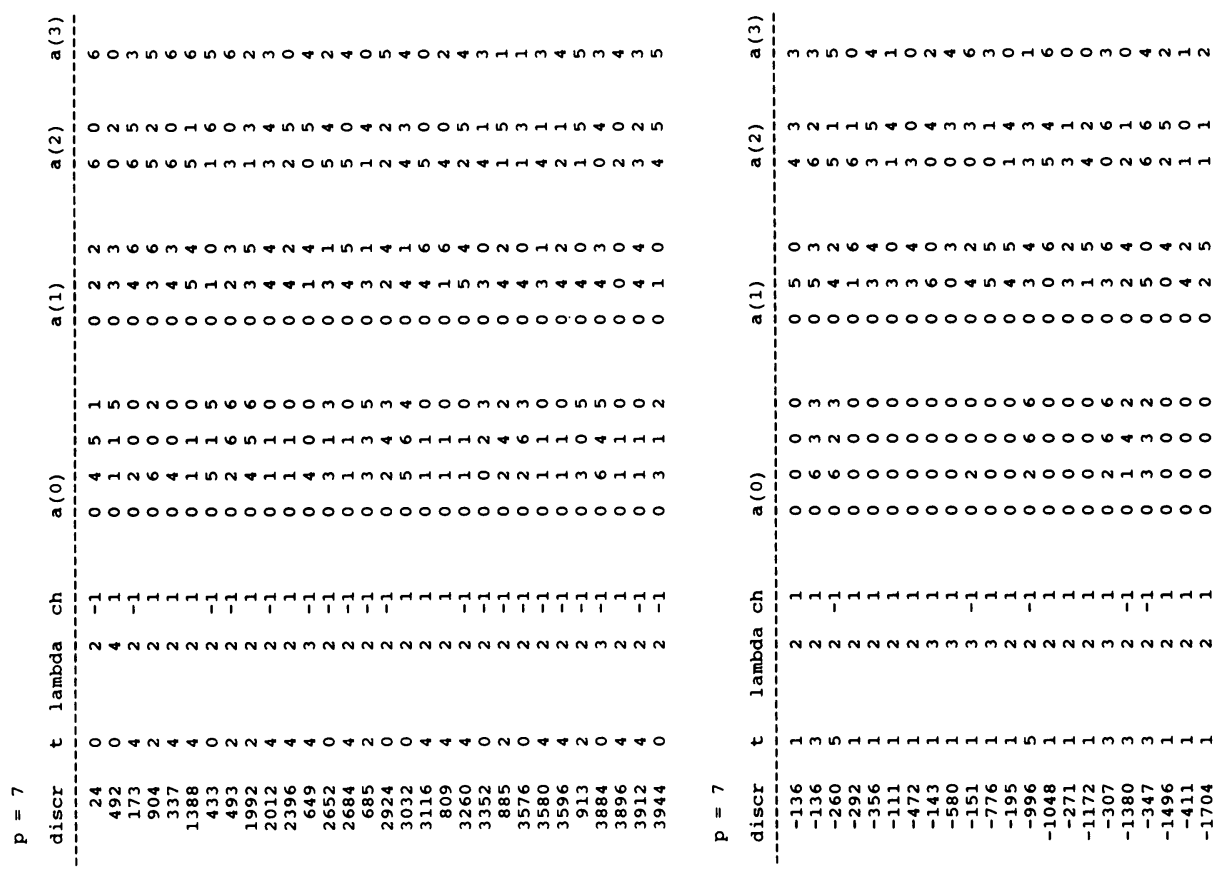

0000000000000000000000000000007000000040000000000000000004000040

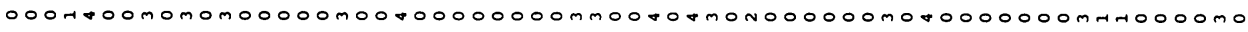

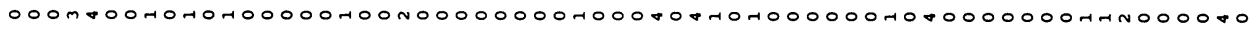
0000000000000000000000000000000000000000000000000000000000000000

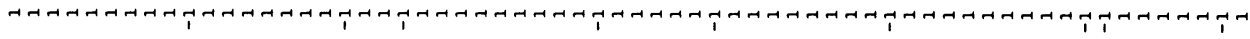

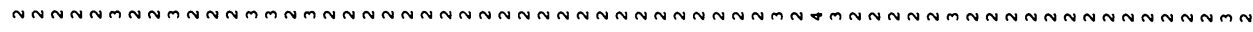




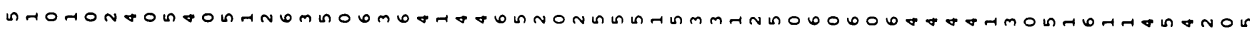

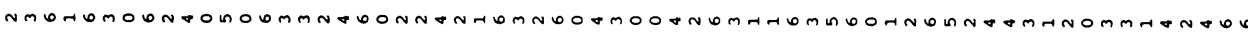

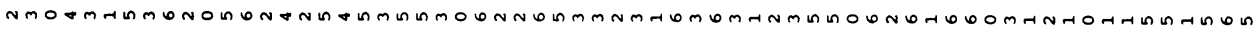

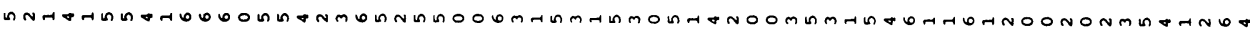

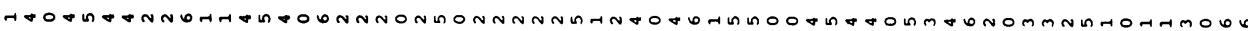
0000000000000000000000000000000000000000000000000000000000000000

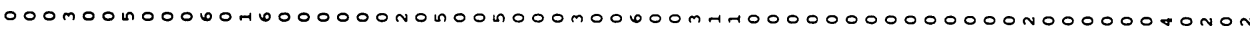

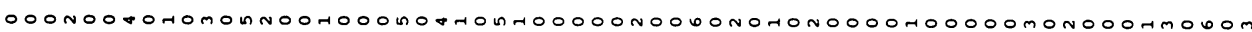

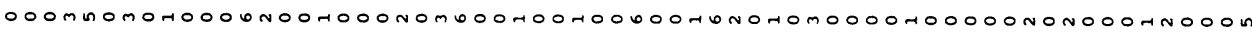
0000000000000000000000000000000000000000000000000000000000000000

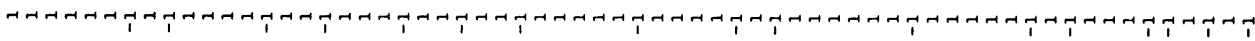

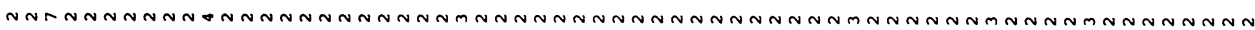

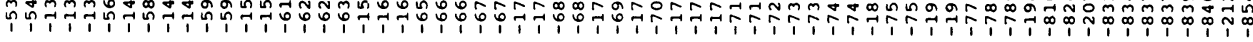

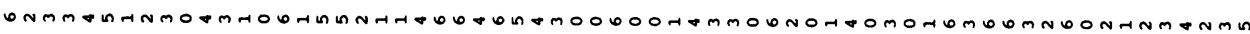
0000000000000000000000000000000000000000000000000000000000000000

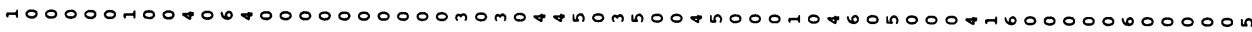

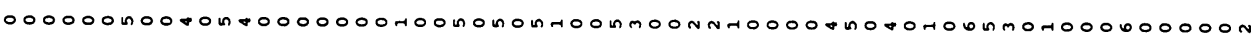

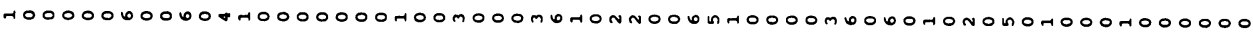
0000000000000000000000000000000000000000000000000000000000000000

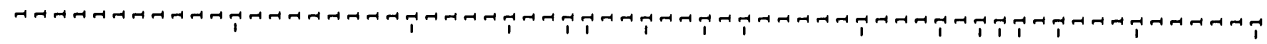

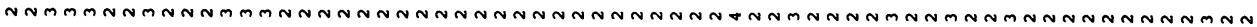




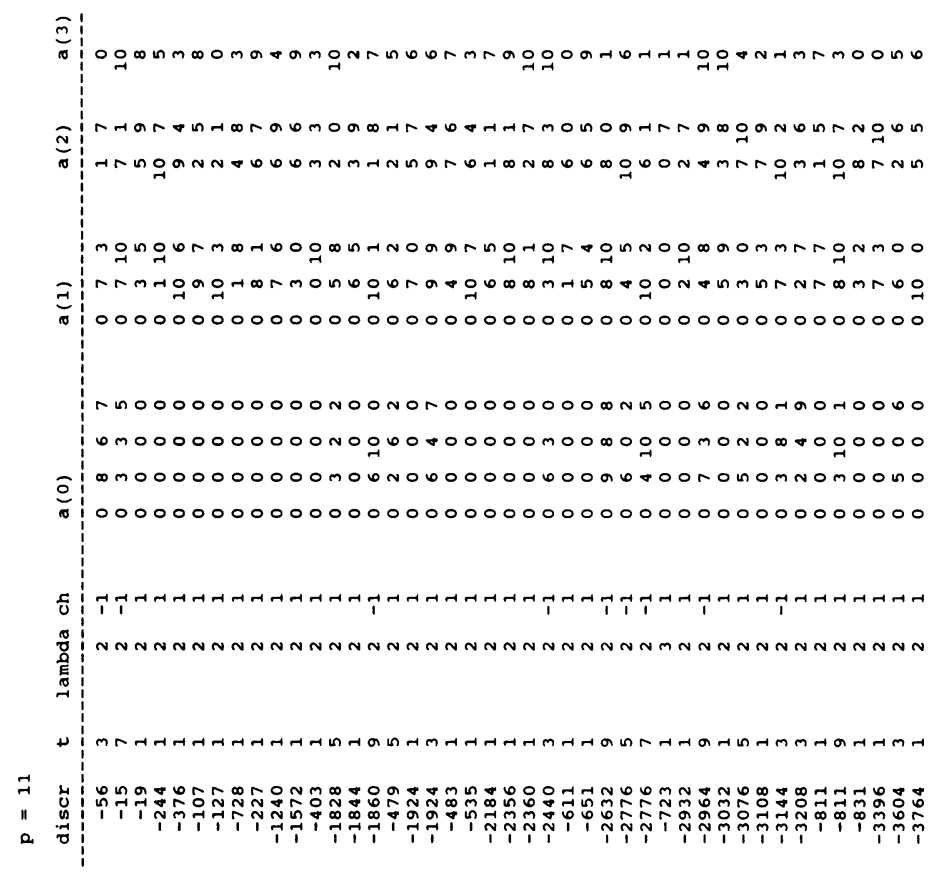

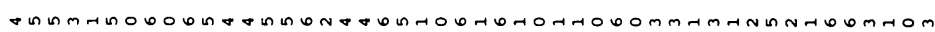

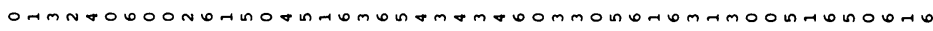

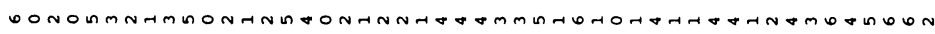

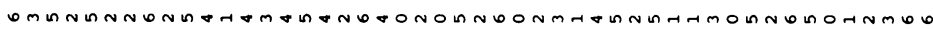

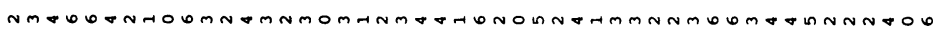
000000000000000000000000000000000000000000000000

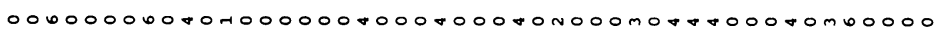

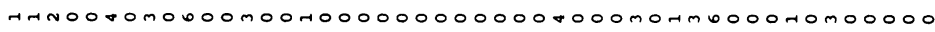

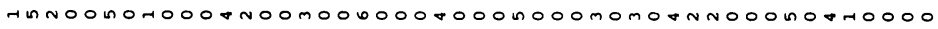
000000000000000000000000000000000000000000000000

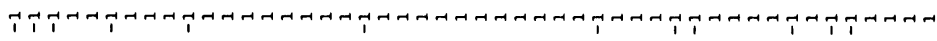

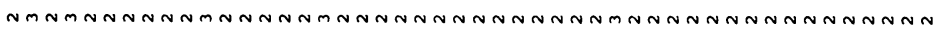

